\title{
Using antibody synergy to engineer a high potency biologic cocktail against $C$. difficile
}

3
Hui Zhao ${ }^{1}$, Michael Tasch ${ }^{1}$, Michael Dodds' ${ }^{1}$, Mesfin Gewe ${ }^{1}$, Anissa Martinez ${ }^{1}$, Melanie Hutton ${ }^{2}$, Kristie Keeney ${ }^{1}$, Alex Pollock ${ }^{1}$, Ben Jester ${ }^{1}$, Nhi Khuong ${ }^{1}$, Mia Zhang ${ }^{1}$, Colin Brady ${ }^{1}$, Mark Heinnickel ${ }^{1}$, Hannah Tabakh ${ }^{1}$, Nathan Sanjaya ${ }^{1}$, Kendra Cruickshank ${ }^{1}$, Troy Paddock ${ }^{1}$, Stacey Ertel $^{1}$, Sarah Struyvenberg ${ }^{1}$, Jason Dang ${ }^{1}$, Tracy Saveria $^{1}$, Chelsea Shanitta ${ }^{1}$, David Fletcher ${ }^{1}$, Lauren Goetsch ${ }^{1}$, Caitlin Gamble ${ }^{1}$, Steven J. Mileto ${ }^{2}$, Ryan Heselpoth ${ }^{3}$, Dena Lyras ${ }^{2}$, Craig A. Behnke $^{1}$, Vincent Fischetti ${ }^{3}$, Brian Finrow ${ }^{1}$, and James M. Roberts ${ }^{1,4}$

${ }^{1}$ Lumen Bioscience, Seattle, WA

${ }^{2}$ Biomedicine Discovery Institute and Department of Microbiology, Monash University, Victoria, Australia ${ }^{3}$ Laboratory of Bacterial Pathogenesis and Immunity, Rockefeller University, New York, NY ${ }^{4}$ Corresponding author: James M. Roberts, Lumen Bioscience, 1441 N. $34^{\text {th }}$ Street, Seattle, WA, 98103,jroberts@lumen.bio

Keywords: Arthrospira platensis; spirulina; C. difficile; VHH; lysin; oral therapeutic; synergy 


\section{ABSTRACT}

22 We applied a mathematical framework originally used to model the effects of multiple

23 inhibitors on enzyme activity to guide the development a therapeutic antibody cocktail, LMN-

24 201, to prevent and treat $C$. difficile infection (CDI). CDI causes hundreds of thousands of cases

25 of severe, often recurrent diarrhea and colitis in the United States annually and is associated

26 with significant morbidity and mortality worldwide. Current therapies for preventing recurrent

$27 \mathrm{CDI}$ are only partially successful, and there are no options available to prevent initial bouts of

28 CDI in at-risk populations. Almost all antibody therapies have been developed and administered

29 as monotherapies. Antibody cocktails are relatively rare even though they have the potential to

30 greatly increase efficacy. One reason for this is our limited understanding of how antibody

31 interactions can enhance potency, which makes it difficult to identify and develop antibodies

32 that can be assembled into optimally effective cocktails. In contrast to the view that antibody

33 synergies depend on unusual instances of cooperativity or allostery, we show that synergistic

34 efficacy requires nothing more than that the antibodies bind independently to distinct epitopes

35 on a common target. Therefore, synergy may be achieved much more readily than is generally

36 appreciated. Due to synergy the LMN-201 antibody cocktail, which targets the $C$. difficile

37 exotoxin $B(T c d B)$, is 300 - to 3000 -fold more potent at neutralizing the most clinically prevalent

38 TcdB toxin types than bezlotoxumab, the only monoclonal antibody currently approved for

39 treatment or prevention of CDI. The efficacy of LMN-201 is further enhanced by inclusion of a

40 phage-derived endolysin that destroys the $C$. difficile bacterium, and which therefore has a

41 complementary mechanism of action to the antibody cocktail. These observations may serve as 
bioRxiv preprint doi: https://doi.org/10.1101/2021.12.21.473715; this version posted January 14, 2022. The copyright holder for this preprint (which was not certified by peer review) is the author/funder, who has granted bioRxiv a license to display the preprint in perpetuity. It is made available under aCC-BY-NC-ND 4.0 International license.

42 a paradigm for the development of high potency biologic cocktails against targets that have

43 proven challenging for single-agent therapies.

44

45 


\section{INTRODUCTION}

47 Clostridioides difficile is a Gram-positive spore-forming anaerobic bacterium ${ }^{1}$. C. difficile

48 infection (CDI) causes severe, recurrent diarrhea and colitis and is associated with significant

49 morbidity and mortality world-wide ${ }^{2}$. According to the U.S. Centers for Disease Control and

50 Prevention, $C$. difficile is the most common cause of health care-associated infections in U.S

51 hospitals, where nearly half a million CDI cases occur each year ${ }^{3}$. Though traditionally

52 considered a nosocomial infection, community-associated CDI is on the rise and now comprises

53 most cases in some regions ${ }^{4}$. Treatment costs are estimated to exceed $\$ 5$ billion a year, but the

54 true economic burden of the disease is far higher when lost quality-adjusted life years are

55 considered $^{4}$.

56 C. difficile virulence is mediated by expression of two homologous, multidomain exotoxins,

$57 \mathrm{TcdA}$ and $\mathrm{TcdB}^{5-8}$. The toxins initially engage cell surface receptors on intestine epithelial cells,

58 which results in internalization by endocytosis ${ }^{9,10}$. Acidification of internalized endosomes

59 triggers a conformational change in the toxin that promotes its translocation into the

60 cytoplasm $^{11}$. Cytoplasmic toxin undergoes autoproteolysis ${ }^{12}$, releasing its $\mathrm{N}$-terminal

61 glucosyltransferase domain that enzymatically inhibits Rho family-GTPases by mono-

62 glucosylation ${ }^{13,14}$. Inactivation of Rho disaggregates the cellular actin cytoskeleton thereby

63 disrupting the colonic epithelium ${ }^{15-17}$, leading to severe inflammation and watery diarrhea

64 characteristic of CDI. The relative importance of TcdA and TcdB for causing C. difficile pathology

65 varies by host species, and current balance of opinion is that human pathophysiology is

66 primarily caused by $\operatorname{TcdB}^{7,18-23}$. 
67 Antibiotic therapy is often successful for initial cases of CDI. However, approximately $20 \%-40 \%$

68 of CDI patients will have a recurrent episode ${ }^{24}$, and the chance of additional episodes in those

69 patients exceeds $40 \%{ }^{25,26}$. Microbial dysbiosis is a consequence of the antibiotics most often

70 used to treat CDI, which creates a niche favorable to C. difficile proliferation ${ }^{27}$ and promotes

71 recurrent disease. Due to side effects and selection for antimicrobial resistance, antibiotics are

72 no longer used prophylactically.

73 One alternative to antibiotics for treatment of microbial pathogens is passive immunization by

74 direct infusion of neutralizing antibodies. Intravenous or intraperitoneal infusion of monoclonal

75 and polyclonal antibodies targeting $T c d A$ and $T c d B$ are protective against primary infection and

76 relapse in $C$. difficile challenge models in mice ${ }^{8,21,28,29}$, hamsters, ${ }^{30,31}$ piglets $^{22,32}$. It is important

77 that immunotherapies used clinically address the natural antigenic variation displayed by

$78 \mathrm{TcdB}^{33}$. Bezlotoxumab, a monoclonal antibody against $\mathrm{TcdB}$, has been adopted for clinical use in

79 conjunction with antibiotics for high-risk patients ${ }^{23,34}$. However, it poorly neutralizes the

80 prevalent TcdB2 variant, and clinically it is only partially effective in reducing recurrent CDI in

81 humans. Oral administration of polyclonal antibodies from cows hyperimmunized with TcdA

82 and TcdB has likewise been evaluated in human trials with reported success but has not

83 progressed to commercial development ${ }^{35-37}$. Most recently, fecal microbiota transplantation

84 (FMT) has also proven effective in reducing recurrence of CDI, presumably by helping to correct

85 the intestinal dysbiosis that predisposes to relapsing disease ${ }^{38}$. Despite these advances there

86 currently are no clinically approved therapeutics to prevent primary CDI. There are no FDA

87 approved CDI vaccines ${ }^{39}$, and the most recent Phase 3 clinical trial of an anti-toxin vaccine was

88 terminated due to futility ${ }^{40}$. 
89 We report here the preclinical development of a four-component therapeutic cocktail to both

90 prevent and treat CDI. The cocktail is comprised of homodimeric versions of three toxin-binding

91 proteins (5D, E3, and 7F) derived from camelid single-domain antibody fragments known as

92 VHHs or nanobodies. Each protein was previously shown to inactivate $\operatorname{TcdB}^{8,28,29,41}$. Binding of

$935 \mathrm{D}$ to $\mathrm{TcdB}$ prevents its $\mathrm{pH}$-driven conformational change that enables pore formation and

94 transit into the cytoplasm from internalised endosomes. E3 binds to the glucosyltransferase

95 domain (GTD) of TcdB, interfering with the autoproteolysis step necessary for enzyme

96 activation. 7F also binds to the GTD, inhibiting catalytic glucosylation/inactivation of Rho-family

97 GTPases by the mature N-terminal glucosyltransferase. The fourth cocktail component is a

98 C. difficile-specific endolysin ${ }^{42}$. These proteins are highly specific, phage-encoded antimicrobial

99 Iytic enzymes that, due their specificity and rapid effect, hold considerable promise for treating

100 bacterial infectious diseases, including $\mathrm{CDI}^{42-44}$. We showed that the selected combination of

101 three dimeric VHHs had a synergistic neutralization potency that vastly exceeded the potency

102 of any single dimeric $\mathrm{VHH}$ and had far greater potency and broader strain coverage than

103 bezlotoxumab ${ }^{23,34}$. We further demonstrated that the therapeutic was enhanced by combining

104 the dimeric VHHs that neutralize TcdB with an endolysin that destroys the $C$ difficile bacterium.

105 LMN-201 was manufactured using the edible microorganism spirulina (Arthrospira platensis) as

106 the expression platform. It was delivered orally as a powder of whole, unrefined spirulina

107 biomass ${ }^{45}$, and is easily scalable, shelf-stable, simple to administer, and broadly neutralizing

108 against all common TcdB types. 


\section{RESULTS}

\section{Spirulina strain engineering}

113 The $C$. difficile therapeutic LMN-201 contained four therapeutic proteins: three homodimeric

114 fusion proteins designated pp1005, pp1006, and pp1007, which incorporated homodimeric

115 derivatives of VHHs 5D, E3 or 7F, respectively; and fourthly, the catalytic domain of the

116 bacteriophage-derived PlyCD endolysin, designated pp1092.

117 The primary structures of each monomer subunit in the VHH homodimers were similar. From

118 amino to carboxy terminus this was: the maltose-binding protein (MBP) chaperone (366 amino

119 acids) ${ }^{46}$, a Gly-Gly-Gly-Gly tetrapeptide linker, a 5HVZ dimerization domain (49 amino acids), a

120 Gly-Ser-Gly-Ser-Gly-Gly peptide linker, a TcdB-binding VHH (111-127 amino acids), a Gly-Ser-Gly

121 tripeptide linker and a hexahistidine tag (543-562 amino acids total) (Figure 1A). The 5HVZ

122 dimerization domain was derived from the mammalian cAMP-dependent protein kinase $\mathrm{A}$

123 (PKA) holoenzyme $47,48$.

124 The endolysin PlyCD has been previously demonstrated to be $C$. difficile specific ${ }^{42-44}$. Full-length

125 PlyCD (pp1093) its catalytic domain alone, PlyCD ${ }_{1-174}(\mathrm{pp} 1092)$, were expressed in spirulina

126 strains sp1286 and SP1287, respectively, with an N-terminal 6x his-tag (Figure 1A).

127 Genes encoding pp1005, pp1006, pp1007, pp1093 and pp1092 were engineered into the

128 spirulina chromosome by homologous recombination (Figure 1B) ${ }^{45}$. The final strains-SP1308,

129 SP1312, SP1313, SP1286 and SP1287, respectively-contained the gene of interest integrated

130 into the spirulina chromosome at a single, pre-selected location and no other exogenous 
131 genetic information. Clonal spirulina filaments were then isolated by micromanipulation,

132 verified as being homozygous with respect to the transgene, and used to create a frozen strain

133 bank (Figure 1B, C)

\section{Characterization of spirulina-expressed anti-TcdB VHHs: expression and binding kinetics}

135 Expression levels of all 5 candidate therapeutic proteins in spirulina were quantified using a

136 capillary electrophoresis-based immunoassay (Figure 2A). After growth in a bioreactor, dried

137 whole SP1308 biomass was comprised of $0.5 \%$ by weight pp1005; SP1312 was comprised of

$1382.3 \%$ pp1006; SP1313 was comprised of $3.4 \%$ pp1007; and SP1287 was comprised of 0.5\%

139 pp1092. At least $90 \%$ of each of these proteins was soluble following a one-hour extraction in

140 an aqueous buffer at neutral $\mathrm{pH}$. The dimeric state of the VHHs on the $5 \mathrm{HVZ}$ scaffold was

141 confirmed by comparing their relative mobilities during size-exclusion chromatography, and by

142 capillary electrophoresis in reducing versus non-reducing conditions (Figure 2B). Intra-scaffold

143 disulfide bonds were present in the extracted proteins (Figure 2B) but were dispensable for

144 stable assembly of the 5HVZ-VHH dimers (Figure 2C).

145 Binding kinetics were measured by biolayer interferometry using recombinant subdomains of

146 TcdB that corresponded to the specific antigen-binding sites of each VHH homodimer

147 (Figure $3 \mathrm{~A}, \mathrm{~B}$ ). The apparent $\mathrm{K}_{\mathrm{D}}$ 's were in the mid- to low-picomolar range. The dimeric versions

148 of all three proteins displayed substantially greater apparent avidity for TcdB than the

149 corresponding monomeric versions (Figure 3B). 


\section{Characterization of TcdB's effect on cell adhesion}

151 Disruption of the actin cytoskeleton by TcdB causes changes in cell shape and cell attachment.

152 In vitro, these cytoskeletal changes can be measured in real-time as a change in impedance

153 using cells cultured on a surface embedded with microelectrodes ${ }^{49,50}$. TcdB1 is the TcdB toxin

154 type most frequently present in worldwide $C$. difficile isolates from infected patients ${ }^{51}$. The

155 source of TcdB1 for these analyses was ribotype (RT) 087 (type strain 10463). The concentration

156 range of TcdB1 tested spanned the amounts reported in human patients with $C$. difficile

157 infection ${ }^{52}$. Data describing TcdB1 toxicity were collected and fit to a four-parameter sigmoid

158 using nonlinear regression to minimize the sums of residuals squared:

$$
\text { Adhesion }=\text { Top }-(\text { Top }- \text { Bottom }) \cdot \frac{T^{H}}{T^{H}+I C_{50}{ }^{H}}
$$

160 where Adhesion indicates the results of the above cell attachment assay; Top and Bottom are

161 the highest (no TcdB1) and lowest (maximal TcdB1) assay values; $T$ is the concentration of

$162 \mathrm{TcdB} 1 ; \mathrm{I} \mathrm{C}_{50}$ is the concentration of TcdB1 eliciting half maximal inhibition in Adhesion; $\mathrm{H}$ is the

163 steepness of the concentration-response relationship.

164 The concentration of half-maximal inhibition of cell adhesion by TcdB1 was $1.268 \mathrm{fM}$ with a Hill

165 slope coefficient of 1.495 (Table 1; Figure 4).

167 Data were collected to evaluate inhibition of TcdB toxicity by each VHH homodimer, and all

168 two- and three-way combinations (Figure 4A; Supplemental Figure 1). Individually, each of the

169 dimeric VHHs had sub-nanomolar $\mathrm{EC}_{50}$ values for TcdB neutralization (Table 1). However, 
170 compared to the single VHHs, the cocktail of the three dimeric VHHs showed remarkable

171 synergy, providing robust neutralization across the very wide range of TcdB concentrations

172 found in patients with $\mathrm{CDI}^{52}$ (Figure 4; Supplemental Table 2). To illustrate, a concentration of

$1731.5 \mathrm{nM}$ of any one of the three dimeric VHHs shifted the IC 50 of TcdB1 10- to 60-fold, whereas a

$1741.5 \mathrm{nM}$ cocktail of all three combined ( $0.5 \mathrm{nM}$ each) shifted the IC 50 of TcdB1 almost 3,000-fold.

175 For TcdB2 the cocktail synergy was similarly striking. At a concentration of $1.5 \mathrm{nM}$ the individual

176 dimeric VHHs shifted the $\mathrm{IC}_{50}$ of $\mathrm{TcdB} 210$ - to 150 -fold, while a $1.5 \mathrm{nM}$ cocktail of the three

177 dimeric $\mathrm{VHHs}\left(0.5 \mathrm{nM}\right.$ each) shifted the $\mathrm{IC}_{50}$ of TcdB2 more than 16,000 -fold.

178 Surprisingly, the dramatic synergy of antibody combinations requires nothing more than that

179 they bind independently to distinct epitopes on the target. Quantitative modeling using

180 measured $\mathrm{K}_{\mathrm{D}}$ 's and the effects of dimeric $\mathrm{VHH}$ mixtures across a large range of TcdB

181 concentrations indicated that this was likely to be the entire explanation for the synergy we

182 observed (Supplemental Text and Supplemental Figures 1,2,3). This suggested that synergy

183 might be much more easily achieved than is generally appreciated, which has important

184 implications for the development of therapeutic antibody cocktails (see Discussion).

\section{Broad coverage of TdcB toxin-types}

186 Mansfield et al. ${ }^{33}$ used C. difficile genomic sequences to define 12 distinct TcdB groups that

187 differed from each other by $3 \%$ or greater. $96 \%$ of the TcdB genes present in that dataset were

188 defined as belonging to groups 1, 2, 3, and 5. Group 1 is the most clinically prevalent group

189 worldwide, and group 2 is expressed by $C$. difficile ribotype 027 , the single most prevalent strain

190 type in the United States, and second most prevalent in Europe $3,51,53,54$. 
191 The high degree of conservation of the pp1005, pp1006, and pp1007 binding sites ${ }^{41}$ suggested

192 that each of the dimeric VHHs would neutralize most, if not all, of the four prevalent TcdB toxin

193 types, except for neutralization of $\mathrm{TcdB}$ group 5 by pp1005. We measured the $\mathrm{IC}_{50}$ values of

194 TcdB types 1.1, 2.1, 3.1, and 5.1 alone and when incubated in 1.5 nM pp1005, pp1006, pp1007,

195 or a cocktail comprised of all three $\mathrm{VHH}$ homodimers ( $0.5 \mathrm{nM}$ of each) and compared these

196 with the neutralizing potency of $3 \mathrm{nM}$ bezlotoxumab (Figure 4). Each VHH homodimer exhibited

197 strong neutralizing activity against all tested toxin types except pp1005, which was only weakly

198 active against toxin type 5 . The triple VHH cocktail synergistically neutralized all tested toxin

199 types, with apparent relative potencies of against each toxin type of $2>1>3>5$. Compared to the

200 triple VHH cocktail, bezlotoxumab was significantly less potent at neutralizing all tested toxin

201 types, and its neutralizing activity against toxin type 2 (ribotype 027) was notably weak.

\section{Characterization of spirulina-expressed anti-C. difficile lysin}

203 PlyCD, a prophage lysin, was found to have a high degree of specificity for degrading $C$. difficile

204 peptidoglycan ${ }^{42}$. The catalytic domain of this protein, $\mathrm{PlyCD}_{1-174}$, efficiently lysed various strains

205 of $C$. difficile while leaving commensal bacteria unaffected, with rare exceptions that included

206 B. subtilis and C. sordellii.

207 Genes encoding pp1093 (full-length lysin PlyCD) and pp1092 (PlyCD $1-174)$ were engineered into

208 spirulina to produce strains SP1286 and SP1287, respectively. Expression of both proteins in

209 spirulina was quantified using a capillary electrophoresis-based immunoassay. After growth in a

210 bioreactor pp1093 comprised $0.1 \%$ of dry biomass and was approximately $50 \%$ soluble

211 following aqueous extraction. pp1092 comprised $0.5 \%$ of total SP1287 biomass, and after 
212 aqueous extraction greater than $90 \%$ of pp1092 was soluble (Figure 5A). Following extraction

213 from SP1286 and SP1287 biomass the pp1093 and pp1092 proteins were found to be

214 monomeric by size-exclusion chromatography and non-reducing PAGE (Figure 5B). However,

215 pp1093 exhibited notable size heterogeneity, with lower molecular size species.

216 The lytic activities of pp1093 and pp1092 were compared in a turbidity reduction assay using

217 extracts from SP1286 and SP1287, and B. subtilis as the target bacterium. In these spirulina cell

218 extracts, the specific activity of pp1092 was 8-fold greater than pp1093 (Figure 5C). The

219 reduced lysin activity of the pp1093 compared to pp1092 was also observed using C. difficile

220 strain ATCC 43255 as the target bacterium (Supplemental Figure 4). The lytic activities of

221 pp1093 and pp1092 isolated from spirulina were not significantly different from the same

222 proteins isolated from E. coli. (Supplemental Figure 4). SP1287 (expressing pp1092) was

223 selected for inclusion in LMN-201 based upon its superior expression level, homogeneity and

224 bioactivity compared to SP1286. As a control for interference, it was shown that pp1092 did not

225 affect toxin neutralization by the anti-TcdB VHH cocktail (Figure 4).

\section{Stabilities of anti-TcdB VHHs and anti-C. difficile lysin}

227 The four therapeutic proteins within LMN-201 all demonstrated resistance to three common

228 intestinal proteases: trypsin, elastase, and chymotrypsin (Figures 6A, 7). When exposed to

229 conditions simulating constitutive levels of these proteases for one hour, pp1005, pp1006, and

230 pp1007 retained nearly 100\% ELISA binding activity compared to undigested protein. At a

231 higher concentration of protease that simulated the induced protease amounts following a

232 meal, pp1005 and pp1006 exhibited some sensitivity to digestion with $0.1 \mathrm{mg} / \mathrm{mL}$ trypsin but 
233 still retained $63 \%$ and $75 \%$ of their antigen-binding activities, respectively. SDS-PAGE and

234 western blotting demonstrated that chymotrypsin cleaved the MBP domain of these dimeric

235 toxin-binding proteins within 30 minutes, leaving the VHH-5HVZ dimer intact. This was

236 consistent with the observation that chymotrypsin did not reduce binding affinity as measured

237 by ELISA, and removal of the MBP moiety may explain the paradoxically increased ability to

238 detect bound VHH in an ELISA after chymotrypsin digestion. To confirm that digestion did not

239 affect bioactivity, chymotrypsin-digested pp1005, pp1006, and p1007 were also evaluated in a

240 cell-adhesion assay. No significant changes in toxin neutralizing activity were observed after

241 exposure to chymotrypsin for at least one hour (Figure 6B).

242 Protease treated pp1092 was analyzed for structural integrity by SDS-PAGE (Figure 7A), and for

243 bioactivity in a turbidity reduction assay using intact $B$. subtilis as the substrate (Figure 7B).

244 pp1092 showed no detectable change in molecular size (25kDa) following digestion with any of

245 the three proteases. In contrast, full length PlyCD $(37 \mathrm{kDa})$ was almost $100 \%$ cleaved to a smaller

$24625 \mathrm{kDa}$ product by all three proteases, consistent with release of the smaller, protease-stable

247 catalytic subdomain. Further, pp1092 retained 100\% bioactivity following digestion with any of

248 the three proteases at the lower level, and greater than $70 \%$ bioactivity following digestion with

249 higher (induced) protease amounts.

\section{In vivo efficacy}

251 Efficacies of cocktail therapeutics containing anti-TcdB VHH homodimers and anti-C. difficile

252 lysins were evaluated in mouse (Figure 8) and hamster (Figure 9) pathogen challenge models. 
253 Cohorts of male C57BL/6J mice were infected with C. difficile spores 24 hours after

254 administration of one of the following treatments by oral gavage: vancomycin (positive

255 control); wild-type spirulina (negative control); Cocktail 1; or Cocktail 2. Cocktail 1 was

256 comprised of equal proportions of spirulina strains SP1308, SP1312, and SP1313 (expressing

257 pp1005, pp1006, and pp1007, respectively) and SP1286 (expressing pp1093). Cocktail 2 was the

258 same but omitted SP1286. Each treatment was then administered once daily following

259 C. difficile challenge.

260 The largest number of $C$. difficile spores was shed by mice treated with wild-type spirulina

261 (Figure 8A). Treatment with Cocktail 1 significantly reduced fecal spore shedding at 24 hours

262 post infection and at the time of euthanasia ( $10^{2.206}$ and $10^{1.867} \mathrm{cfu} / \mathrm{g}$ lower, respectively), than

263 wild-type spirulina controls and significant by t-test with $p=0.0175$ and $p<0.001$ (Supplemental

264 Table 3). Fewer spores were observed shed by animals treated with only the toxin-neutralizing

265 antibodies (Cocktail 2), but the difference did not reach statistical significance at either

266 timepoint.

267 The largest maximum weight loss was observed in the negative control group, mice treated

268 with wild-type spirulina, $3.922 \mathrm{~g}$ (Figure 8B; Supplemental Table 4). Treatment with either

269 spirulina cocktail or vancomycin all reduced the maximum weight loss significantly. On average,

270 the maximum weight lost by an individual treated with Cocktail 1 was $2.057 \mathrm{~g}$ less than the

271 group treated with wild-type spirulina and significant by t-test with $p<0.01$. The maximum

272 weight lost by subjects treated with Cocktail 2 was $2.102 \mathrm{~g}$, a result significant by t-test with

$273 p<0.001$ 
274 Treatment with the spirulina therapeutic Cocktails 1 and 2 also increased survival rates, with

275 borderline significance possibly due to small sample size (Figure 8C). None of the mice treated

276 with Cocktail 1 perished due to $C$. difficile-related causes $(4 / 4,100 \%$ survival) which was

277 equivalent to the vancomycin-treated group (10/10, 100\% survival). The increased survival rate

278 was borderline significant relative to the wild-type spirulina-treated group by log-rank test with

$279 p=0.079$. The group treated with Cocktail 2 also exhibited higher survival rates than the

280 negative control $(6 / 7,87 \%$ compared to $2 / 5,40 \%)$ with borderline significance by log-rank test

281 with $p=0.14$. The mouse efficacy study suggests that a spirulina anti-TcdB cocktail

282 supplemented with the lysin is effective at reducing bacterial load and improving clinical

283 outcomes in vivo.

284 The Golden Syrian Hamster model of CDI has been used previously for evaluating orally

285 delivered antibodies against $C$. difficile toxins ${ }^{30}$. The experimental groups we used were

286 Cohort 1: no treatment, Cohort 2: wild-type spirulina, Cohort 3: anti-TcdB VHHs (3) + Anti-TcdA

287 VHHs (2) + lysin PlyCD, all delivered within whole spray-dried spirulina biomass. Each treatment

288 was administered twice daily by oral gavage, from days -1 through day 12 . It was necessary to

289 include anti-TcdA VHHs in this protocol because TcdA contributes significantly to disease in

290 hamsters (unlike in humans) ${ }^{8}$. We observed that the therapeutic protein cocktail significantly

291 extended survival time compared to the wild-type spirulina control $(p=0.015)$ (Figure 9A), and

292 significantly reduced $C$. difficile colonization of the cecum by more than an order of magnitude

293 when compared to untreated or wild-type treated groups ( $p=0.046$ and $p=0.0041$, respectively)

294 (Figure 9B). 


\section{DISCUSSION}

296 We report here the first use of quantitative analyses of drug synergy for preclinical and clinical

297 development of a therapeutic antibody cocktail. These analyses build on a general framework

298 for evaluating the effects of multiple drugs acting on a single target that was initially developed

299 to model enzyme inhibition ${ }^{55}$. In that context it was shown that mutually exclusive inhibitors

300 (i.e., competitive) have an additive inhibitory effect, whereas mutually nonexclusive inhibitors

301 (i.e., independent) have a multiplicative (i.e., synergistic) effect. This framework can be applied

302 more generally, for example to antibody inhibitors of a protein ${ }^{56}$, and has the potential to

303 inform decisions around drug cocktail formulation, including selection of lead candidates and

304 optimization of their relative concentrations.

305 Synergy is usually not considered in the development of cocktail therapeutics. To some degree

306 this is due to the costs and complexities associated with developing drug combinations, but it is

307 also because such synergies are often thought to be rare, arising from cooperative or unusual

308 allosteric effects ${ }^{57}$. A small number of anti-viral therapeutics have been intentionally developed

309 as antibody cocktails ${ }^{58,59}$, but this was done to reduce the likelihood of pathogen escape.

310 Moreover, if drug concentrations are only evaluated within a 2- or 3-fold range of their

311 respective $\mathrm{EC}_{50}$ values, synergy can be difficult to distinguish from simple additivity, and is

312 therefore easily overlooked. Accordingly, almost all clinically approved antibody cocktails were

313 first developed as solo therapeutics, and subsequently only a small subset have been

314 empirically evaluated in combination, often without regard to understanding whether

315 underlying synergies might have contributed to improved efficacy. 
316 In contrast to the prevailing view that synergy is rare and elusive we have demonstrated here,

317 using the specific example of anti-C. difficile toxin antibodies, that synergy can simply be the

318 result of the laws of mass action governing the independent interactions of drugs with a

319 common target, and therefore may be more readily achieved than is generally thought.

320 Moreover, the synergistic efficacy of independently acting drugs increases nonlinearly with

321 concentration and can be enormous when concentrations substantially exceed the $\mathrm{EC}_{50}$. We

322 emphasize that this is not a complex function of allostery or cooperativity, but a simple

323 consequence of independent Michaelis-Menten binding. This has general and important

324 implications for therapeutic development. Synergistically acting antibodies will be particularly

325 useful for treating a disease like CDI, where single antibody therapeutics struggle to achieve the

326 very high level of target inhibition required. That the cocktail also offers broader strain

327 coverage and protection against escape variants adds to its appeal.

328 Beyond infectious diseases, antibody synergy may also be important for treating diseases with

329 complex etiologies where single-agent interventions have only been moderately successful, for

330 example inflammatory diseases of the gastrointestinal tract and cardiometabolic disease. The

331 results here offer antibody drug developers a paradigm for developing high potency cocktail-

332 based interventions against diseases refractory to single-antibody approaches.

$333 \mathrm{CDI}$ is an appealing initial target for clinically evaluating these principles, because current

334 therapies for preventing recurrent CDI are only partially successful, and there are no options

335 available to prevent initial bouts of CDI in at-risk populations. LMN-201 is a spirulina-expressed

336 and orally delivered cocktail of four therapeutic proteins that target both the C. difficile 
337 bacterium and the key driver of its virulence, exotoxin TcdB. Orally delivered protein

338 therapeutics may offer significant advantages over their injected counterparts for treatment

339 and prevention of recurrent CDI. Oral administration can deliver high amounts of therapeutic

340 proteins directly to the intestine epithelium, whereas systemically delivered antibodies have

341 poor partitioning coefficients from the vasculature to the gut lumen ${ }^{60}$. Moreover, the risk of off-

342 target toxicity is reduced: the therapeutic proteins within LMN-201 are too large for intact

343 systemic absorption ${ }^{45}$. At least three prior clinical trials have reported safe and efficacious oral

344 administration of bovine antibodies against $C$. difficile toxins derived from milk or colostrum ${ }^{35-}$

345 , supporting the feasibility of this approach.

346 The harsh environment of the gastrointestinal tract is considered challenging for orally

347 delivered protein therapeutics. LMN-201 addresses this challenge in several ways. First, the

348 therapeutic proteins in LMN-201 showed significant resistance to the major GI proteases.

349 Second, for human administration the product is delivered orally in polymer-coated capsules

350 designed to release the drug product in the ileum. Third, spirulina-based cGMP manufacturing

351 is highly scalable, making feasible cost-effective administration of the drug product throughout

352 the period of high risk for CDI recurrence, even if the harsh GI environment necessitates daily or

353 twice daily administration to maintain therapeutic levels at the site of action ${ }^{42}$.

354 Perhaps of greatest significance is the extraordinary potency of LMN-201: each dimeric VHH

355 within LMN-201 had a sub-nanomolar EC 50 for TcdB inhibition, and this was substantially

356 enhanced by cocktail formulation. An oral dose of 1,500 mg of spirulina biomass (500 $\mathrm{mg}$ of

357 each VHH-spirulina strain) will deliver to the intestine a combined dimeric VHH concentration of 
358 approximately $2.6 \mu \mathrm{M}$, which is more than 1,000-fold higher than minimally required ${ }^{61,62}$.

359 Pharmacokinetic data from a Phase 1 trial of LMN-201 confirmed that the amount of bioactive

360 VHH recovered in the distal ileum following oral administration was more than sufficient to

361 neutralize TcdB-levels previously reported in patients at risk for CDI recurrence (manuscript in

362 preparation).

363 The fourth LMN-201 component-a bacteriophage-derived endolysin-not only broadens

364 C. difficile strain coverage but also enhances efficacy. While TcdB is the key virulence factor in

$365 \mathrm{CDI}$, approximately $5 \%$ of patients are infected by $C$. difficile strains that secrete TcdA but not

$366 \mathrm{TcdB}^{52}$. Other virulence factors are also associated with $\mathrm{CDI}$, including the $C$. difficile binary

367 toxin (CDT), which is associated with increased 30-day mortality ${ }^{63}$. Inclusion of the lysin may

368 offer protection against these non-canonical $C$. difficile strains, as well as novel strains with new

369 toxin variants that might emerge in the future.

370 The lysin may also increase therapeutic potency in at least two ways. First, clinical performance

371 is augmented by the lysin's previously demonstrated synergy with standard-of-care

372 vancomycin, which act in concert to disrupt $C$. difficile cell wall structure ${ }^{42}$. Second, these

373 agents independently intervene at two steps in the pathway that controls virulence- $C$. difficile

374 proliferation and toxin action on the gastrointestinal epithelium. The lysin thus complements

375 the VHH homodimers with a potential for orthogonal synergy to enhance LMN-201's clinical

376 real-world effectiveness. 


\section{MATERIALS AND METHODS}

\section{Engineering of spirulina}

380 Markerless spirulina strains were generated as described ${ }^{45}$. In brief, the natural spirulina gene

381 WP_006618409.1, which confers resistance to kanamycin, was replaced with an exogenous

382 gene encoding resistance to streptomycin to generate spirulina strain SP205. SP205 was then

383 transformed with constructs containing the gene of interest, WP_006618409.1, as well as the

384 homology arms. The transformants were selected with kanamycin. Single filaments were

385 isolated and propagated to generate cell banks. Cells were then thawed from the cell banks and

386 DNA was extracted to be used to confirm complete segregation and verify sequence of the

387 WP_006618409.1 via PCR and Sanger Sequencing.

\section{Illumina sequencing of spirulina}

389 Spirulina genomic DNA was extracted using a Blood and Tissue Kit (Qiagen, 69504) with minor

390 modifications. Spirulina biomass was resuspended in buffer ATL. It was digested with

391 proteinase $\mathrm{K}(2 \mathrm{mg} / \mathrm{mL})$ overnight at $56{ }^{\circ} \mathrm{C}$ and then treated with RNase $\mathrm{A}(10 \mathrm{mg} / \mathrm{mL})$ for

39220 minutes at $56^{\circ} \mathrm{C} .400 \mu \mathrm{L}$ of buffer $\mathrm{AL}$ was added and the biomass was pelleted for 10

393 minutes at $13,000 \mathrm{~g}$. The supernatant was then transferred to a fresh tube with $250 \mu \mathrm{L}$ of $100 \%$

394 ethanol, mixed and applied to DNeasy columns. The samples were washed once with $500 \mu \mathrm{L}$

395 buffer AW1 and once with $500 \mu \mathrm{L}$ AW2 and eluted using molecular grade water. The amount of

396 genomic DNA was quantified by a Qubit dsDNA HS Kit (Thermo, Q33230) and prepared for

397 Illumina analysis using the Illumina DNA prep kit (Illumina, 20018704). Illumina sequencing of 
398 the spirulina genome was performed on a MiSeq instrument using a MiSeq Reagent Kit v2

399 (Illumina, MS-102-2003).

400 Illumina dataset analysis for copy number determination

401 Copy numbers of the genes of interest in SP1287, SP1308, SP1312, and SP1313 were

402 determined by comparing expression of the transgenes to 10 randomly selected genes

403 (referred to as "normalization genes": $p p a X, \operatorname{rps} A, \operatorname{ch} / M$, $\operatorname{deg} U 2, \operatorname{radA}, \operatorname{rbcL}, \operatorname{sdhA}, \operatorname{psbC}, \operatorname{cys} A 1$,

404 smc6) in the genome. A copy number of about 1 , within plus/minus 0.15 , was expected and set

405 as the benchmark for a full genomic integration of 1 gene per genome copy. As a validation

406 control, we also chose a second set of 10 random spirulina genes (thrB1, hisC1, isfD2, pilA, purB,

$407 a p c E, n b / A, g p m B$, bicA2, $c p c B A$ ) which we compared pairwise to the "normalization genes" set

408 in a manner identical to the transgene. Gene copy number per genome was determined by first

409 using samtools (http://www.htslib.org/doc/) to generate an mpileup file with Illumina reads per

410 base pair. Then, the median read per base pair was determined across the 10 "normalization

411 genes" and the transgene. The median read per base pair of the transgene was then divided by

412 the 10 "normalization genes" median read-per-base-pair value.

\section{Preparation of spirulina extracts for analysis of soluble protein}

414 Soluble extracts from spray-dried or paste samples were prepared by bead beating in a

415 Percellys evolution bead beater (Bertin Technologies). Beads for this procedure were purchased

416 in pre-packed tubes from MP-Bio (cat\# 6911500). Dried spirulina biomass was resuspended in

417 PBS containing Pierce Protease Inhibitor minitablets and $1 \mathrm{mM}$ PMSF at a biomass

418 concentration of $2-10 \mathrm{mg} / \mathrm{mL}$. Biomass samples were then centrifuged at $15,000 \mathrm{~g}$ at $4{ }^{\circ} \mathrm{C}$ for 30 
419 minutes, and the soluble fraction was transferred to a separate tube for downstream

420 applications.

\section{Expression analysis of soluble recombinant proteins in spirulina}

422 Recombinant protein expression in spirulina was measured by capillary electrophoresis

423 immunoassay (CEIA) using a Jess system (ProteinSimple). The Jess system was run as

424 recommended by the manufacturer. Briefly, dried biomass samples were diluted to a

425 concentration of $0.1-1 \mathrm{mg} / \mathrm{mL}$ using water and a $5 \mathrm{X}$ master mix prepared from an EZ Standard

426 Pack 1 (Bio-Techne). Purified protein controls used to generate standard curves were typically

427 loaded at a range of concentrations from 0.25-8 $\mu \mathrm{g} / \mathrm{mL}$. A 12-230 kDa Jess/Wes Separation

428 Module (ProteinSimple) was used for separation. A mouse anti-His-Tag antibody (GenScript)

429 was diluted 1:100 and used as the primary antibody. An anti-mouse NIR fluorescence-

430 conjugated secondary antibody (ProteinSimple) was primarily used for detection. Non-reducing

431 denaturing gels were performed in the same manner; however, reductant was withheld from

432 the 5X master mix. Data analysis was performed using the Protein Simple Compass software

433 and Microsoft Excel.

434 Expression analysis of total recombinant proteins (soluble and insoluble) in spirulina

435 Spray dried or paste samples were prepared by bead beating as above. The samples were

436 solubilized in PBS buffer mentioned above with a Triton X-100 concentration at $0.02 \%$.

437 Following lysis, samples were diluted to $0.1-1 \mathrm{mg} / \mathrm{mL}$ in extraction buffer $(60 \mathrm{mM}$ dithiothreitol,

$43860 \mathrm{mM} \mathrm{Na}_{2} \mathrm{CO}_{3}, 6 \mathrm{mM}$ ethylenediamine tetraacetic acid, 2\% (w/v) sodium dodecyl sulfate,

$43912 \%(w / v)$ sucrose and $0.02 \%$ Triton X-100). After the addition of the lysis buffer, samples were 
440 immediately heated at $100{ }^{\circ} \mathrm{C}$ for 120 seconds, then centrifuged at $16,000 \mathrm{~g}$ for 5 minutes.

441 Extracted samples were displayed on the Jess system as described above.

\section{Cell lines, bacterial strains, toxins, and antibodies}

443 Vero cells (ATCC) were cultured in Dulbecco's Modified Eagle Medium (DMEM) supplemented

444 with $10 \%$ heat-inactivated fetal bovine serum and $1 \%$ penicillin-streptomycin (Gibco). The cells

445 were grown in a high-humidity incubator at $37{ }^{\circ} \mathrm{C}$ and $5 \% \mathrm{CO}_{2}$. C. difficile toxin TcdB-10463 was

446 expressed in B. megaterium (a gift from C. Shoemaker, Tufts University) and purified using $\mathrm{Ni}$ -

447 NTA affinity and ion exchange chromatography. Purified C. difficile toxins TcdB-027, TcdB-1470,

448 and TcdB-078 were purchased from tgcBIOMICS. Bezlotoxumab was sourced from Henry Schein

449 Medical.

\section{Expression of TcdB fragments in bacterial systems}

451 The $\mathrm{VHH}$-binding domains on $\mathrm{TcdB}^{41}$ were designed for bacterial expression with an $\mathrm{N}$-terminal

452 hexahistidine tag followed by MBP and TEV protease recognition site (ENLYFQG) and C-terminal

453 Avi-tag (LNDIFEAQKIEWHE) for enzymatic biotinylation. Based on the structural information, we

454 designed the 5D-binding region, the delivery and receptor-binding domain (DRBD) of TcdB for

455 expression using residues from amino acid position 1092 to position 1433 of TcdB. VHHs 7F and

456 E3 bind regions on the glucosyltransferase domain (GTD). A fragment containing this region was

457 designed to include amino acid positions 2 through amino acid 543 of TcdB. The constructs

458 were subcloned into a modified $\mathrm{pET} 28 \mathrm{~b}(+)$ vector where the Kanamycin bacterial resistance

459 gene was replaced with Ampicillin bacterial resistance gene. Constructs were sequence verified

460 by Sanger sequencing (Genewiz). Sequence verified plasmids were transformed into BL21(DE3) 
461 Escherichia coli cells (New England Biolabs) and plated on LB medium supplemented with 100

$462 \mu \mathrm{g} \mathrm{mL} \mathrm{L}^{-1}$ ampicillin. Protein was expressed using autoinduction protocol ${ }^{64}$. Briefly, $1 \mathrm{~L}$ of Super

463 Broth media containing appropriate selection drug was inoculated with overnight cultures from

464 individual transformants, shaken at $37{ }^{\circ} \mathrm{C}$ for 8 hours followed by $16^{\circ} \mathrm{C}$ for 24 hours. Expression

465 cultures were pelleted by centrifugation and stored at $-20{ }^{\circ} \mathrm{C}$ until purification. Frozen cell

466 pellets were thawed on ice and resuspended in $100 \mathrm{~mL}$ of $50 \mathrm{mM}$ Tris $\mathrm{pH} 8.0,300 \mathrm{mM} \mathrm{NaCl}$

467 supplemented with pierce protease inhibitor tablets EDTA free (Thermo Fisher Scientific cat. no.

468 A32965) and $1 \mathrm{mM}$ phenylmethylsulfonyl fluoride (PMSF) (Thermo Fisher Scientific cat. no.

469 36978). Cells were lysed by high pressure homogenization (HPH) (Microfluidics LM20) at 12,000

470 psi. The lysates were clarified by centrifugation at $18,500 \mathrm{~g}$ at $4{ }^{\circ} \mathrm{C}$ for 30 minutes using tabletop

471 centrifuge (Eppendorf Centrifuge 5810R). Soluble protein was separated from cell debris and

472 the supernatant was passed through $5 \mathrm{~mL}$ HisTrap columns (Cytiva, cat. no. 17524802)

473 equilibrated with 50mM Tris $\mathrm{pH} 8.0,300 \mathrm{mM} \mathrm{NaCl}$ and $20 \mathrm{mM}$ imidazole (Thermo Fisher

474 Scientific, cat. no. 03196-500). His-tagged protein bound HisTrap columns were washed with 10

475 column volumes (CV) wash buffer (50 mM Tris, pH 8.0, $300 \mathrm{mM} \mathrm{NaCl}$ and $20 \mathrm{mM}$ imidazole).

476 Bound his-tagged protein were eluted using gradient flow at slope of $10 \%-100 \%$ elution buffer

477 (50 mM Tris $\mathrm{pH}$ 8.0, $300 \mathrm{mM} \mathrm{NaCl}$ and $250 \mathrm{mM}$ imidazole) in $5 \mathrm{CV}$. Fractions containing the

478 eluted protein were assessed by SDS-PAGE gel. SDS-PAGE analyses were performed by

479 incubating $2 \mu \mathrm{L}$ of purified protein elution in NuPAGE LDS Sample Buffer (Thermo Fisher

480 Scientific) at $70^{\circ} \mathrm{C}$ for 10 minutes. Bis-Tris NuPAGE 4 to $12 \%$ gels were placed into gel box filled

481 with NuPAGE MES SDS Buffer. Samples were loaded and gels run at constant volt of $170 \mathrm{mV}$ for

48240 minutes, followed by staining using Coomassie Blue gel stain. Fractions were pooled based 
483 on level of purity, concentrated, and buffer exchanged into PBS. The samples were then filtered

484 through a $0.2 \mu \mathrm{m}$ filter and run over a size-exclusion column on AKTA Pure FPLC-based size-

485 exclusion chromatography HiLoad 16/600 Superdex 200 pg column (Cytiva, cat. no. 28989335)

486 equilibrated in PBS. Purity and final analysis were performed using SDS-PAGE gel where $3 \mu \mathrm{g}$

487 purified protein samples were incubated at $70^{\circ} \mathrm{C}$ in LDS loading buffer with or without $20 \mathrm{mM}$

488 dithiothreitol (DTT) reducing agent (Thermo Fisher Scientific, cat. no. R0862). Protein samples

489 were separated by size on NuPAGE Bis-Tris gels as described above. Purified samples were

490 biotinylated using BirA biotin ligase (Avidity Inc.) at $1 \mu \mathrm{g}$ of BirA enzyme per $10 \mathrm{nM}$ of protein.

\section{Protein purification from spirulina biomass}

492 The TcdB-binding proteins developed from VHHs 5D (pp1005, batch no. 145), E3 (pp1006, batch

493 no. 147), and 7F (pp1007, batch no. 147), and full-length lysin PlyCD (pp1092, batch no. 025)

494 were expressed in spirulina and purified from spray-dried biomass using Ni-NTA affinity and

495 size-exclusion chromatography 2-step purification protocol. Dried spirulina biomass from

496 strains expressing heterologous proteins were rehydrated at $30 \mathrm{~mL} / \mathrm{g}$ of biomass in phosphate

497 buffer saline lysis buffer supplemented with protease inhibitor mix of $1 \mathrm{mM}$

498 phenylmethylsulfonyl fluoride (PMSF), (Thermo Fisher Scientific cat. no. 36978) and Pierce

499 Protease Inhibitor Tablets, EDTA-free, (Thermo Fisher Scientific cat no A32965). Rehydrated and

500 homogenous biomass resuspension were lysed using a high-pressure homogenizer

501 (Microfluidics LM20) at 12,000 psi. The lysates were clarified by centrifugation at $18500 \mathrm{~g}$ on

502 tabletop Centrifuge (Eppendorf Centrifuge 5810R) for 30 minutes. Supernatant were clarified

503 by filtration using a coffee filter followed by $0.22 \mu \mathrm{m}$ membrane filter. Recombinant proteins

504 were purified from the clarified lysate by affinity chromatography as describe above using $5 \mathrm{~mL}$ 
505 HisTrap columns (Cytiva, cat. no. 17524802). Hexa-Histidine tagged and HisTrap-purified

506 samples were further refined using AKTA Pure FPLC-based size-exclusion chromatography on

507 HiLoad 16/600 Superdex 200 pg column (Cytiva, cat. no. 28989335). Protein purity and sizing

508 were assayed by size-exclusion chromatography on analytical Superdex 200 increase 10/300

509 (Cytiva, cat. no. 28990944) where $100 \mu \mathrm{L}$ of purified protein at $\sim 1 \mathrm{mg} / \mathrm{mL}$ was run in PBS.

510 Purified protein samples were also assessed on SDS-PAGE under reducing and non-reducing

511 conditions. $3 \mu \mathrm{g}$ protein samples were incubated at $70{ }^{\circ} \mathrm{C}$ in LDS loading buffer with or without

$51220 \mathrm{mM}$ dithiothreitol (DTT) reducing agent (Thermo Fisher Scientific, cat. no. R0862). Proteins

513 were determined to be $>90 \%$ pure and exhibited the expected molecular weight size

514 distribution.

\section{$515 \quad$ Native gel analysis}

516 To assess the importance of disulfide bonds in pp1005, pp1006, and pp1007 dimer formation,

517 we compared protein migration in native and SDS-PAGE gel under reduced and non-reduced

518 conditions. Native gel analysis was performed following the manufacturer's recommendation

519 (Life Technologies) where $1 \mu \mathrm{g}$ of purified protein was mixed with Native Page Sample Buffer

520 supplemented with or without 20 mM DTT. Native page anode buffer was prepared by mixing

$52150 \mathrm{~mL}$ NativePage $20 \mathrm{x}$ running buffer and $950 \mathrm{~mL}$ single distilled water. Similarly, $1 \mathrm{x}$

522 NativePage Dark Blue Cathode buffer was prepared by mixing $50 \mathrm{~mL}$ NativePage 20 x Running

523 buffer with $50 \mathrm{~mL}$ NativePage 20X Cathode Additive and $900 \mathrm{~mL}$ single distilled water. Gel

524 boxes were set up on ice and samples were loaded prior to adding running buffer. Inner

525 chamber of gel box was filled with NativePage Dark Blue Cathode buffer while the outer 
526 chamber was filled with NativePage Anode buffer. Gels were run at a constant $150 \mathrm{~V}$ for 2.5

527 hours. Comparable sample preparations were run in SDS-PAGE gel as described above.

\section{Assessment of VHH binding to TcdB fragments with bio-layer interferometry (BLI)}

529 BLI measurements were performed on the Octet RED96 system (ForteBio) using High Precision

530 Streptavidin (SAX) Biosensors (ForteBio). Biosensors were hydrated with phosphate buffer,

$531 \mathrm{pH} 7.4$ at room temperature for 10 minutes on Microplate 96-well flat-bottom (Greiner,

532 655209). All kinetics experiments were performed at $30{ }^{\circ} \mathrm{C}$ temperature with $500 \mathrm{rpm}$ agitation

533 in the kinetics module. Biosensors were dipped into PBS containing wells for 60 seconds prior to

534 antigen loading. Biosensors were loaded with enzymatically biotinylated TcdB antigen

535 fragments at $10 \mu \mathrm{g} / \mathrm{mL}$ in phosphate buffer, $\mathrm{pH} 7.4$ for 200 seconds to achieve $\sim 0.6-1 \mathrm{~nm}$

536 response. Loading was quenched by incubating biosensors in $50 \mu \mathrm{M}$ Biocytin (Sigma Aldrich,

$537576-19-2$ ) for 60 seconds. Baseline were established by incubating antigen loaded biosensors in

538 kinetics buffer (PBS + $0.02 \%$ Tween 20, $0.1 \%$ BSA, $0.05 \%$ sodium azide) for 300 seconds. After

539 stablishing baseline, the rate of association was measured by incubating antigen loaded

540 biosensor tips into 3-fold dilution series of monomeric (pp1239, pp1238, and pp1240

541 respectively) or dimeric (pp1005, pp1006, and pp1007 respectively) 5D, E3, and 7F constructs.

542 The concentration series used to determine binding kinetics of pp1238 were $93.6 \mathrm{nM}, 31.2 \mathrm{nM}$,

$54310.4 \mathrm{nM}, 3.47 \mathrm{nM}, 1.16 \mathrm{nM}$, and $0.36 \mathrm{nM}$. Concentrations used to determine binding kinetics of

544 pp1239 were $93.6 \mathrm{nM}, 31.2 \mathrm{nM}, 10.4 \mathrm{nM}, 3.47 \mathrm{nM}, 0.38 \mathrm{nM}$, and $0.38 \mathrm{nM}$. Concentrations used

545 for pp1240 were $92.2 \mathrm{nM}, 30.7 \mathrm{nM}, 10.2 \mathrm{nM}, 3.41 \mathrm{nM}, 1.14 \mathrm{nM}$ and $0.38 \mathrm{nM}$. pp1005 was

546 assayed at $16.2 \mathrm{nM}, 5.39 \mathrm{nM}, 1.8 \mathrm{nM}, 0.60 \mathrm{nM}, 0.20 \mathrm{nM}$ and $0.07 \mathrm{nM}$. Concentrations used for

547 pp1006 were $16.8 \mathrm{nM}, 5.6 \mathrm{nM}, 1.87 \mathrm{nM}, 0.62 \mathrm{nM}, 0.21 \mathrm{nM}$, and $0.07 \mathrm{nM}$. pp1007 were assayed 
at concentrations of $84.9 \mathrm{nM}, 28.3 \mathrm{nM}, 9.43 \mathrm{nM}, 3.14 \mathrm{nM}, 1.05 \mathrm{nM}$, and $0.35 \mathrm{nM}$. Analyte

549 bound biosensors were dipped into kinetics buffer for 300 - 600 seconds to measure rate of

550 dissociation. Kinetic analysis was performed using the HT 11.1.1.39 Data Analysis module

551 (ForteBio). Results were double referenced. Both association and dissociation steps were used

552 in 1:1 binding model global data fitting model.

\section{Toxin-neutralization assay}

554 Toxin-neutralization assays using TcdB1.1, TcdB2.1, TcdB3.1 and TcdB5.1 were performed using

555 the Agilent xCELLigence RTCA MP. This system measures cell adherence via biosensors in the

556 bottom of a 96-well plate by applying a small electric current across microelectrodes. A unitless

557 measurement called cell index $(\mathrm{Cl})$ was then calculated based on changes in electrode

558 impedance. Because adherent cells increase electrode impedance, toxin induced cell rounding

559 can be determined from changes in $\mathrm{Cl}$.

560 Agilent 96-well E-plates were seeded with $100 \mu \mathrm{l} /$ well of $1 \times 10^{5}$ cells $/ \mathrm{mL}$ in DMEM culture

561 medium with 10\% FBS and 1\% Pen/Strep and allowed to rest for 30 minutes before going into

562 an xCELLigence real-time cell analyzer in a Caron $\mathrm{CO}_{2} 37^{\circ} \mathrm{C}$ incubator to run overnight. $\mathrm{Cl}$ was

563 monitored every 15 minutes for 20 to 24 hours. TcdB was serially diluted in culture medium in a

$56496-$-well set up plate. The VHH homodimer, lysin, and bezlotoxumab samples were diluted in

565 medium and then added to the serially diluted toxin. The TcdB/protein mixture was incubated

566 at $37^{\circ} \mathrm{C}$ for 1 hour. After 1 hour, media was carefully removed from the cells seeded onto the E-

567 plates and $100 \mu \mathrm{L}$ of the $\mathrm{VHH}$ homodimer and toxin mixture was added to the cells in each well.

$568 \mathrm{Cl}$ was monitored every 15 minutes for at least 24 hours. Each run included a TcdB only 
569 standard curve as a positive control and cells only as a negative control. VHH homodimer was

570 also tested in the absence of TcdB to assess any effects of $\mathrm{VHH}$ alone on $\mathrm{Cl}$. At the 24-hour time

571 point after treatment, the $\mathrm{Cl}$ measurements for each well were normalized by setting the $\mathrm{Cl}$ to

5721 at the last reading taken before treatment was added. The normalized cell index (nCl) was

573 plotted using Prism and a nonlinear regression analysis was performed to calculate the $\mathrm{IC}_{50}$ of

574 TcdB under treatments.

575 TcdB's effect on cell adhesion and inhibition of TcdB by anti-TcdB VHH homodimers

576 Cell adhesion assay data were imported, normalized, and analyzed using R (version 4.1.1 The R

577 Foundation for Statistical Computing). Nonlinear regression was performed using the

578 minpack.Im routine in the nIsLM package, which implements the Levenberg-Marquardt

579 algorithm to perform bounded minimization ${ }^{65}$.

\section{Lysin activity assay}

581 The lytic activity of pp1092 against Bacillus subtilis SL4 was assessed based upon previously

582 described methods with modifications to adapt the assay to a 96-well plate format ${ }^{42}$. Mid-log

583 phase grown SL4 were grown in $\mathrm{BHI}\left(37^{\circ} \mathrm{C}, 250 \mathrm{rpm}\right)$ harvested and washed twice in PBS by

584 centrifugation (room temperature, 6,000g, 10 minutes). Bacteria were then combined with 1ug

585 digested or undigested pp1092 in a final volume of $200 \mu \mathrm{L}(\mathrm{OD}=1.0)$ and then turbidity

586 reduction was measured over time $\left(37^{\circ} \mathrm{C}\right.$, shaking). Each unknown was fit along with 5 3-fold

587 serial dilutions of undigested standards using a modified 5-parameter nonlinear logistic model.

588 The time of $50 \% \mathrm{OD}_{600}, \mathrm{TC}_{50}$, was estimated as a 4-parameter nonlinear logistic model with lysin

589 concentration as the independent variable. For the standards, this concentration is known. For 
590 the unknown sample, this value is estimated. All results were expressed as the mean \pm standard

591 deviation as calculated by Excel software (v16.48, Renton, WA). Standard curves were

592 determined by linear regression analysis by Excel software (v16.48, Renton, WA). EC 50 values

593 were calculated using GraphPad Prism (version number, GraphPad Software Inc, San Diego, CA).

594 For comparison of spirulina-expressed and E. coli-expressed PlyCD, C. difficile strain ATCC 43255

595 was grown to mid-log phase (optical density at $\left.600 \mathrm{~nm}\left(\mathrm{OD}_{600 \mathrm{~nm}}\right)=0.5\right)$ at $37^{\circ} \mathrm{C}$ under

596 anaerobic conditions in reduced Brain Heart Infusion Medium (BD Biosciences) supplemented

597 with $0.5 \%(\mathrm{wt} / \mathrm{vol})$ yeast extract and $0.1 \%(\mathrm{wt} / \mathrm{vol}) \mathrm{L}$-cysteine. The bacteria were harvested at

5984,000 rpm for 15 minutes, washed and resuspended to an OD $_{600 \mathrm{~nm}}$ of $2.0 \mathrm{in} 50 \mathrm{mM}$ sodium

599 phosphate, $\mathrm{pH}$ 7.0. Using a 96-well nontreated polystyrene microtiter plate (Corning), $100 \mu \mathrm{L}$ of

600 buffer with or without (untreated controls) lysin at initial concentrations ranging from 4 to 256

$601 \mu \mathrm{g} / \mathrm{mL}$ were added to individual wells. Following the addition of $100 \mu \mathrm{L}$ bacteria, the $\mathrm{OD}_{600 \mathrm{~nm}}$ of

602 each sample was measured every 60 seconds for a total of 15 minutes at $37^{\circ} \mathrm{C}$ using a

603 SpectraMax M5 microplate reader (Molecular Devices). All data represents the average of

604 triplicate experiments.

\section{VHH homodimer protease digestion for ELISA and neutralization assays}

606 In vitro protease digestions of anti-C. difficile VHH homodimers were prepared in a matrix of

607 soluble lysate from wild-type spirulina (SP003) in Bis-Tris buffer pH 6.0 and treated with trypsin,

608 chymotrypsin, or elastase. VHH homodimers in PBS at were diluted in SP3 lysate, Bis-Tris buffer,

609 and protease so that the final concentrations were VHH homodimer, $20 \mathrm{mg} / \mathrm{mL}$ of SP3 lysate,

610 and a "fed" or "fasted" amount of protease. This level of SP3 lysate and VHH homodimer 
611 simulates a expression level of $1 \%$ in spirulina (by dry weight). For trypsin and chymotrypsin,

612 the "fed" and "fasted" levels of protease in the digestion reactions were 0.1 and $0.01 \mathrm{mg} / \mathrm{mL}$,

613 while for elastase, 5 and $0.5 \mu \mathrm{g} / \mathrm{mL}$ were used respectively. Samples were incubated at $37^{\circ} \mathrm{C}$ for

6141 hour with shaking at $900 \mathrm{rpm}$ in an Eppendorf ThermoMixer. At the end of the incubation,

615 reactions were quenched with an equal volume of stop buffer containing 2 mM PMSF and 2x

616 Pierce protease inhibitor minitablet (Thermo Fisher) in PBS; elastase digests were additionally

617 neutralized with a $1 \mathrm{mM}$ elastase inhibitor III. Samples were kept on ice until preparation for

618 ELISA or cell rounding assay. The final concentration of each VHH homodimer after protease

619 neutralization was $0.1 \mathrm{mg} / \mathrm{mL}$.

\section{VHH homodimer quantitation by ELISA}

621 Digested VHH homodimers were analyzed for binding activity via ELISA using TcdB. Thermo

622 Scientific Nunc ELISA plates were coated with $100 \mu \mathrm{L} /$ well of inactive TcdB at $2 \mu \mathrm{g} / \mathrm{mL}$ in CBC

623 binding buffer and incubated overnight at $4{ }^{\circ} \mathrm{C}$. Plates were washed 3 times with PBS

624 supplemented with 0.05\% Tween-20 (PBS-tw), then blocked for 1 hour with $300 \mu \mathrm{L} /$ well of

625 block (PB-tw supplemented with 5\% BLOTTO). Dilutions of undigested and digested VHH

626 homodimer were prepared in a low-binding 96-well plate by serially diluting with block. 6

627 dilutions of each undigested $\mathrm{VHH}$ homodimer were prepared for a standard curve. Two

628 dilutions of each protease digested sample were prepared for quantification by interpolation

629 from the standard curve. A $100 \mu \mathrm{L}$ sample of each VHH homodimer was added to ELISA plate

630 wells and incubated for 1 hour at room temperature on a plate shaker. The plates were washed

631 as before. An HRP-conjugated anti-camelid VHH antibody cocktail (Genscript) was diluted

63250,000 -fold in block and $100 \mu \mathrm{L} /$ well was added to the ELISA plates for 50 minutes with shaking. 
633 Plates were washed 2 times with PBS-tw and 1 time with PBS. Finally, $100 \mu \mathrm{L} /$ well of TMB Ultra

634 ELISA substrate (Thermo Fisher) was added to each well. The plates were developed in the dark

635 at room temperature on a plate shaker for 10 minutes before being quenched with $50 \mu \mathrm{L}$ of 2.5

636 M sulfuric acid, and the absorbance was read at $450 \mathrm{~nm}$ with a Spectra Max M5 plate reader.

637 All digestion reactions were in duplicate, in 3 independent experiments.

\section{Calculation of VHH homodimer protease resistance}

639 The concentration of $\mathrm{VHH}$ homodimer in the protease-digested samples was calculated by

640 interpolating the concentration from standard curves generated with undigested $\mathrm{VHH}$

641 homodimer. The percentage of remaining VHH homodimer activity after digestion was

642 calculated by dividing the interpolated value by the amount present if no $\mathrm{VHH}$ homodimer were

643 digested. Each replicate was calculated separately, and the average and standard deviation

644 were determined across all replicates.

\section{Toxin neutralization assay of digested VHH homodimer s}

646 Chymotrypsin-digested pp1005, pp1006, and pp1007 were assessed for toxin-neutralizing

647 activity as described. The final concentration of pp1005, pp1006, and pp1007 was $0.05 \mathrm{nM}$,

$6485 \mathrm{nM}$, and $5 \mathrm{nM}$ respectively and the TcdB was $1: 5$ serially diluted starting at $250 \mathrm{pM}$.

\section{Lysin digestion conditions}

650 In digestion buffer (20 mM Tris, $150 \mathrm{mM} \mathrm{NaCl}, 3 \mathrm{mM} \mathrm{CaCl} 2$ at $\mathrm{pH}$ 6.4) $0.2 \mu \mathrm{g} / \mu \mathrm{L}$ protein was

651 mixed with proteases trypsin (Promega sequencing grade), chymotrypsin (BD), and elastase

$652(\mathrm{BD})$ at $0.1 \mu \mathrm{g} / \mu \mathrm{L}$ or $0.01 \mu \mathrm{g} / \mu \mathrm{L}$ and incubated for 1 hour at $37^{\circ} \mathrm{C}$ (200 rpm shaking). Reactions

653 were immediately quenched by addition of $1 \mathrm{mM}$ PMSF and Protease Inhibitor Mini Tablets, 
654 EDTA-free (Thermo Scientific, Pierce). For samples with elastase, $1 \mathrm{mM}$ elastase inhibitor III

655 (Milipore Sigma) was added as well. To visualize digestion, $2 \mu \mathrm{g}$ of digested and undigested

656 proteins were displayed a gradient BOLT-PAGE gel at $200 \mathrm{~V}$ for 20 minutes and then stained

657 with Coomassie.

\section{Densitometry}

659 Pixel intensity of Coomassie stained proteins were measured using ImageJ software,

660 normalizing all bands to the undigested band for each condition after subtracting background

661 pixel intensity of an identically sized bounding box.

\section{Preparation of $C$. difficile spores for mouse study}

663 C. difficile strain M7404, a ribotype 027 clinical isolate from Canada, was cultured on HIS-T agar

$664(3.7 \%(\mathrm{w} / \mathrm{v})$ heart infusion $(\mathrm{HI})(\mathrm{Oxoid}), 0.5 \%(\mathrm{w} / \mathrm{v})$ yeast extract, $1.5 \%(\mathrm{w} / \mathrm{v})$ agar

665 supplemented with $0.375 \%(\mathrm{w} / \mathrm{v})$ glucose, $0.1 \%(\mathrm{w} / \mathrm{v})$ L-cysteine and $0.1 \%(\mathrm{w} / \mathrm{v})$ sodium

666 taurocholate (New Zealand Pharmaceuticals)) in an anaerobic chamber (Don Whitley Scientific)

667 with an atmosphere of $10 \% \mathrm{H} 2,10 \% \mathrm{CO}_{2}$ and $80 \% \mathrm{~N} 2$ at $37^{\circ} \mathrm{C}$. To prepare spores, strains were

668 sub-cultured on HIS-T agar and inoculated into $500 \mathrm{~mL}$ tryptone yeast (TY) broth (3.0\%

669 tryptone, $2.0 \%$ yeast extract, and $0.1 \%$ sodium thioglycolate) and grown anaerobically at $37{ }^{\circ} \mathrm{C}$

670 for 10 days. Spores were harvested by centrifugation at $10,000 \mathrm{~g}$ for 20 minutes at $4{ }^{\circ} \mathrm{C}$ and

671 washed 5 times with chilled distilled water prior to heat-shocking at $65^{\circ} \mathrm{C}$ for 20 minutes and

672 quantified by plating onto HIS-T agar. Spores were adjusted to $10^{6} \mathrm{CFU} / \mathrm{mL}$ in PBS containing

$6730.05 \%$ Tween-80 in preparation for mouse infections. 


\section{Mouse study design}

675 Animal handling and experimentation was performed in accordance with Victorian State

676 Government regulations and approved by the Monash University Animal Ethics Committee

677 (Monash University AEC no. 21731). Male C57BL/6J mice, 6-7 weeks old, were assigned to

678 either a spirulina therapeutic treatment group, positive control (vancomycin-treated), or

679 negative control (wild-type spirulina-treated) group and caged in groups of 5. To induce

680 susceptibility to $C$. difficile, the animals were pre-treated with an antibiotic cocktail in the

681 drinking water containing kanamycin $(0.4 \mathrm{mg} / \mathrm{mL})$, gentamicin $(0.035 \mathrm{mg} / \mathrm{mL})$, colistin $(850$

$682 \mathrm{U} / \mathrm{mL})$, metronidazole $(0.215 \mathrm{mg} / \mathrm{mL})$, vancomycin $(0.045 \mathrm{mg} / \mathrm{mL})$, and cefaclor $(0.3 \mathrm{mg} / \mathrm{mL})$ for

6837 days, followed by 4 days of cefaclor $(0.3 \mathrm{mg} / \mathrm{mL})$ alone. Starting 1 day prior to infection (day -

684 1), the animals were orally gavaged with $200 \mu \mathrm{L}$ of treatment once daily for the first two days

685 (peak of infection) and then $100 \mu \mathrm{L}$ once daily for the remainder of the study. Mice were

686 infected with $10^{5}$ spores/mouse by oral gavage.

687 The spirulina treatments and controls were resuspended in PBS ( $5 \% \mathrm{w} / \mathrm{v})$ by mixing up and

688 down using an $18 \mathrm{G}$ blunt needle and $1 \mathrm{~mL}$ syringe for 2.5 minutes and administered within 10

689 minutes of preparation. Vancomycin was diluted to $6.25 \mathrm{mg} / \mathrm{mL}$ prior to administration. On the

690 day of $C$. difficile infection (day 0), antibiotic pre-treatment was ceased, the mice were switched

691 to plain water, and infection was initiated 3 hours after the treatment dose. Mice were

692 monitored daily for signs of infection, including weight loss, and were euthanized upon losing

$69315 \%$ body weight, if they reached pre-defined clinical endpoints as required by ethics, or at the

694 end of the study (day 4). As a surrogate assessment of colonization, fecal samples collected at

69524 hours post-infection and then again at the time of euthanasia were analyzed to enumerate 
696 the shedding of $C$. difficile spores. Subjects treated with a spirulina therapeutic cocktail were

697 compared to the cohort administered wild-type spirulina.

\section{Quantification of spore shedding from mouse feces}

699 Fecal pellets were collected 24 hours post-infection and at time of euthanasia, resuspended in

700 PBS $(100 \mathrm{mg} / \mathrm{mL})$, heat shocked $\left(30\right.$ minutes, $65^{\circ} \mathrm{C}$ ) and plated for spore enumeration onto HIS

701 agar supplemented with $0.1 \%(w / v)$ L-cysteine, $0.1 \%(w / v)$ sodium taurocholate, $0.375 \%(w / v)$

702 glucose, $250 \mu \mathrm{g} / \mathrm{mL}$ D-cycloserine, $8 \mu \mathrm{g} / \mathrm{mL}$ cefoxitin, and $10 \mu \mathrm{g} / \mathrm{mL}$ erythromycin.

703 Analysis of colonization data was performed by fitting linear models of $\log _{10} \mathrm{cfu} / \mathrm{g}$ data per

704 landmark time with treatment as a (potential) explanatory factor. A coefficient for wild-type

705 spirulina describes the mean $\log _{10} \mathrm{cfu} / \mathrm{g}$ for that treatment, and coefficients for each other

706 treatment describes the difference in mean $\log _{10} \mathrm{cfu} / \mathrm{g}$ for that treatment relative to wild-type

707 spirulina. Standard errors of estimates were reported, along with t-statistics and 2-tailed p-

708 value corresponding to the t-statistic with $\alpha=0.05$.

\section{Analysis of clinical outcomes-mouse study}

710 Analysis of maximum weight loss data was performed by fitting linear models of maximum

711 weight loss data with treatment as a (potential) explanatory factor. A coefficient for wild-type

712 spirulina describes the mean maximum weight loss for that treatment, and coefficients for each

713 other treatment describes the difference in mean loss for that treatment relative to wild-type

714 spirulina. Standard errors of estimates were reported, along with t-statistics and 2-tailed p-

715 value corresponding to the t-statistic with $\alpha=0.05$. 
716 Analysis of survival data was performed on tabulated number at risk and number of events at

717 each time. Data was compared for each treatment to wild-type spirulina results and a $\mathrm{P}$ value

718 was computed using the log-rank statistic for each comparison.

719 Hamster study challenge agent preparation and confirmation

720 C. difficile strain R20291 (NAP1/BI/027) spore stock was titered on TCCFA plates after a 30-

721 minute incubation at $65{ }^{\circ} \mathrm{C}$ and determined to be $5 \times 10^{5} \mathrm{cfu} / \mathrm{mL}$ in a volume of $5 \mathrm{~mL}$ of PBS.

722 This spore stock was diluted to 15,000 spores/50 $\mu \mathrm{L}$ and further diluted in an equal volume of

723 sterile PBS containing 5\% sucrose to facilitate administration of 15,000 spores in $100 \mu \mathrm{L}$ per

724 animal.

725 To verify challenge doses, $100 \mu \mathrm{L}$ of inoculum were serially diluted (10-fold dilutions) and plated

726 onto TCCFA plates. After incubation of the plates under anaerobic conditions at $37^{\circ} \mathrm{C}$ for 48

727 hours, the number of colonies were enumerated, and the doses calculated based on these

728 numbers. The inoculum was measured to be 15,000 cfu.

\section{Hamster study design}

730 Hamsters (Envigo, Indianapolis, IN) were visibly checked upon arrival and allowed to acclimate

731 to their new environment for 2 days. Each animal was given a physical exam and randomly

732 assigned (adjusting only to distribute body weight evenly) to 1 group. They were fed ad libitum

733 with sterilized 2018S Teklad Global 18\% Rodent Diet (Envigo, Indianapolis, IN) and sterile water

734 provided in standard bottles with sippers during the study. To sensitize the animals to C. difficile

735 infection, clindamycin, $15 \mathrm{mg} / \mathrm{mL}$ in saline, was administered intraperitoneally at $30 \mathrm{mg} / \mathrm{kg}$,

736 once a day, for 3 consecutive days. During clindamycin treatment, cage bottoms were changed 
bioRxiv preprint doi: https://doi.org/10.1101/2021.12.21.473715; this version posted January 14,2022 . The copyright holder for this preprint (which was not certified by peer review) is the author/funder, who has granted bioRxiv a license to display the preprint in perpetuity. It is made available under aCC-BY-NC-ND 4.0 International license.

737 daily for 3 consecutive days beginning on the first day to prevent bacterial recolonization from

738 coprophagy. 


\section{ACKNOWLEDGEMENTS}

740 We thank Charles Shoemaker, George McDonald, and Fred Cross for help, discussion, and

741 advice. We thank Meagan James and other members of the Lyras laboratory for help with the

742 mouse studies. For the hamster challenge studies Lumen Bioscience has utilized the non-clinical

743 and pre-clinical services program offered by the National Institute of Allergy and Infectious

744 Diseases, especially support from Sangun Lee, Christian Gonzalez and Ryan Ranallo. 


\section{REFERENCES}

1. Knight, D. R., Elliott, B., Chang, B. J., Perkins, T. T. \& Riley, T. V. Diversity and Evolution in the Genome of Clostridium difficile. Clinical Microbiology Reviews 28, 721-741 (2015).

2. Balsells, E. et al. Global burden of Clostridium difficile infections: a systematic review and meta-analysis. Journal of Global Health 9, 010407 (2019).

3. Guh, A. Y. et al. Trends in U.S. Burden of Clostridioides difficile Infection and Outcomes. N. Engl. J. Med. 382, 1320-1330 (2020). versus standard of care alone for the prevention of recurrent Clostridium difficile Infection in High-Risk Patients in Spain Adv. Ther (2018) doi:doi.org/10.1007/s12325-018-0813-y.

5. Seal, D. et al. Treatment of relapsing Clostridium difficile diarrhoea by administration of a non-toxigenic strain. Eur J Clin Microbiol 6, 51-53 (1987). 467, 711-713 (2010).

7. Lyras, D. et al. Toxin B is essential for virulence of Clostridium difficile. Nature 458, 11767631179 (2009).

764 8. Chen, K. et al. A probiotic yeast-based immunotherapy against Clostridioides difficile 765 infection. Sci Transl Med 12, eaax4905 (2020).

766 9. Tao, L. et al. Frizzled proteins are colonic epithelial receptors for $C$. difficile toxin B. Nature $767538,350-355$ (2016).

768 10. Henkel, D. et al. Receptor Binding Domains of TcdB from Clostridioides difficile for 769 Chondroitin Sulfate Proteoglycan-4 and Frizzled Proteins Are Functionally Independent and 770 Additive. Toxins (Basel) 12, 736 (2020).

771 11. Qa'Dan, M., Spyres, L. M. \& Ballard, J. D. pH-induced conformational changes in Clostridium 772 difficile toxin B. Infection and Immunity 68, 2470-2474 (2000).

773 12. Reineke, J. et al. Autocatalytic cleavage of Clostridium difficile toxin B. Nature 446, 415-419 774 (2007).

775 13. Sehr, P. et al. Glucosylation and ADP ribosylation of rho proteins: effects on nucleotide 776 binding, GTPase activity, and effector coupling. Biochemistry 37, 5296-5304 (1998). 
14. Just, I. et al. Glucosylation of Rho proteins by Clostridium difficile toxin B. Nature 375, 500778503 (1995).

15. Aelst, L. V. \& Symons, M. Role of Rho family GTPases in epithelial morphogenesis. Genes \& Development 16, 1032-1054 (2002). GTPases in intestinal epithelial barrier regulation in vitro. J Cell Physiol 226, 1196-1203 (2011).

18. Carter, G. P. et al. Defining the Roles of TcdA and TcdB in Localized Gastrointestinal Disease, Systemic Organ Damage, and the Host Response during Clostridium difficile Infections. mBio 6, e00551 (2015).

19. Mileto, S. J. et al. Clostridioides difficile infection damages colonic stem cells via TcdB, impairing epithelial repair and recovery from disease. Proceedings of the National Academy of Sciences 117, 8064-8073 (2020).

791 20. Bella, S. D., Ascenzi, P., Siarakas, S., Petrosillo, N. \& Masi, A. di. Clostridium difficile Toxins A 792 and B: Insights into Pathogenic Properties and Extraintestinal Effects. Toxins (Basel) 8, 134-25 793 (2016).

794 21. Hutton, M. L. et al. Bovine antibodies targeting primary and recurrent Clostridium difficile 795 disease are a potent antibiotic alternative. Sci. Rep. 7, 16020-13 (2017).

799 23. Wilcox, M. H. et al. Bezlotoxumab for Prevention of Recurrent Clostridium difficile Infection. 800 N. Engl. J. Med. 376, 305-317 (2017).

801 24. Dieterle, M. G., Rao, K. \& Young, V. B. Novel therapies and preventative strategies for 802 primary and recurrent Clostridium difficile infections. Annals of the New York Academy of 803 Sciences 1435, 110-138 (2019).

804 25. Song, J. H. \& Kim, Y.-S. Recurrent Clostridium difficile Infection: Risk Factors, Treatment, and 805 Prevention. Gut Liver 13, 16-24 (2019).

806 26. Singh, T. et al. Updates in Treatment of Recurrent Clostridium difficile Infection. J Clin Med 807 Res 11, 465-471 (2019). 
27. Pruss, K. M. \& Sonnenburg, J. L. C. difficile exploits a host metabolite produced during toxinmediated disease. Nature 593, 261-265 (2021).

28. Yang, Z. et al. A novel multivalent, single-domain antibody targeting TcdA and TcdB prevents fulminant Clostridium difficile infection in mice. J. Infect. Dis. 210, 964-972 (2014). Outcome in Three Animal Models of Clostridium difficile Infection. Clinical and Vaccine Immunology 23, 774-784 (2016).

815 30. Roberts, A. K. et al. A Novel, Orally Delivered Antibody Therapy and Its Potential to Prevent 816 Clostridioides difficile Infection in Pre-clinical Models. Front Microbiol 11, 578903 (2020).

817 31. Babcock, G. J. et al. Human Monoclonal Antibodies Directed against Toxins A and B Prevent 818 Clostridium difficile-Induced Mortality in Hamsters. Infection and Immunity 74, 6339-6347 819 (2006).

820 32. Sponseller, J. K. et al. Hyperimmune Bovine Colostrum as a Novel Therapy to Combat 821 Clostridium difficile Infection. J. Infect. Dis. 5, 549-8 (2014).

822 33. Mansfield, M. J. et al. Phylogenomics of 8,839 Clostridioides difficile genomes reveals 823 recombination-driven evolution and diversification of toxin A and B. PLoS Pathog 16, e1009181 824 (2020).

825 34. Bartlett, J. G. Bezlotoxumab - A New Agent for Clostridium difficile Infection. N. Engl. J. 826 Med. 376, 381-382 (2017).

827 35. Dissel, J. T. van et al. Bovine antibody-enriched whey to aid in the prevention of a relapse of 828 Clostridium difficile-associated diarrhoea: preclinical and preliminary clinical data. J Med 829 Microbiol 54, 197-205 (2005). difficile-associated diarrhoea: bovine anti-Clostridium difficile whey protein to help aid the 832 prevention of relapses. Gut 56, 888 (2007).

833 37. Mattila, E. et al. A randomized, double-blind study comparing Clostridium difficile immune 834 whey and metronidazole for recurrent Clostridium difficile-associated diarrhoea: Efficacy and 835 safety data of a prematurely interrupted trial. Scand J Infect Dis 40, 702-708 (2009). after Clostridioides difficile infection: Lessons Learned from a Phase 2 Trial. Clin Infect Dis 313, 8381719 (2020). 
839 39. Ghose, C. \& Kelly, C. P. The Prospect for Vaccines to Prevent Clostridium difficile Infection. 840 Infectious Disease Clinics of North America 29, 145-162 (2015).

841 40. Bruyn, G. de et al. Safety, immunogenicity, and efficacy of a Clostridioides difficile toxoid 842 vaccine candidate: a phase 3 multicentre, observer-blind, randomised, controlled trial. The 843 Lancet Infectious Diseases 21, 252-262 (2021).

844 41. Chen, P. et al. Structure of the full-length Clostridium difficile toxin B. Nat Struct Mol Biol 26, 845 712-719 (2019).

846 42. Wang, Q., Euler, C. W., Delaune, A. \& Fischetti, V. A. Using a Novel Lysin To Help Control 847 Clostridium difficile Infections. Antimicrobial Agents and Chemotherapy 59, 7447-7457 (2015).

848 43. Fischetti, V. A. Bacteriophage lysins as effective antibacterials. Current Opinion in 849 Microbiology 11, 393-400 (2008).

850 44. Peng, Z. et al. A Novel Bacteriophage Lysin-Human Defensin Fusion Protein Is Effective in 851 Treatment of Clostridioides difficile Infection in Mice. Front. Microbio. 9, 3234 (2018).

852 45. Jester, B. et al. Expression and manufacturing of protein therapeutics in spirulina. bioRxiv 8532021.01 .25 .427910 (2021) doi:10.1101/2021.01.25.427910.

854 46. Raran-Kurussi, S. \& Waugh, D. S. The Ability to Enhance the Solubility of Its Fusion Partners 855 Is an Intrinsic Property of Maltose-Binding Protein but Their Folding Is Either Spontaneous or 856 Chaperone-Mediated. Plos One 7, e49589 (2012).

857 47. Hanoune, J. \& Defer, N. Regulation and role of adenylyl cyclase isoforms. Annu. Rev. 858 Pharmacol. Toxicol. 41, 145-174 (2001).

859 48. Burgers, P. P. et al. Structure of smAKAP and its regulation by PKA-mediated 860 phosphorylation. FEBS J 283, 2132-2148 (2016).

861 49. Ryder, A. B. et al. Assessment of Clostridium difficile Infections by Quantitative Detection of 862 tcdB Toxin by Use of a Real-Time Cell Analysis System. J Clin Microbiol 48, 4129-4134 (2010).

863 50. Pollock, N. R. Ultrasensitive Detection and Quantification of Toxins for Optimized Diagnosis 864 of Clostridium difficile Infection. Journal of Clinical Microbiology 54, 259-264 (2016).

865 51. Shen, E. et al. Subtyping analysis reveals new variants and accelerated evolution of 866 Clostridioides difficile toxin B. Commun Biology 3, 347 (2020).

867 52. Alonso, C. D. et al. Ultrasensitive and quantitative toxin measurement correlates with 868 baseline severity, severe outcomes, and recurrence among hospitalized patients with 869 Clostridioides difficile infection. Clin Infect Dis ciab826- (2021) doi:10.1093/cid/ciab826. 
870 53. Tickler, I. A. et al. Changes in molecular epidemiology and antimicrobial resistance profiles 871 of Clostridioides (Clostridium) difficile strains in the United States between 2011 and 2017.

872 Anaerobe 60, 102050 (2019).

873 54. Li, Z. et al. Ribotype Classification of Clostridioides difficile Isolates Is Not Predictive of the 874 Amino Acid Sequence Diversity of the Toxin Virulence Factors TcdA and TcdB. Front Microbiol 87511,1310 (2020).

876 55. Chou, T. C. \& Talaly, P. A simple generalized equation for the analysis of multiple inhibitions 877 of Michaelis-Menten kinetic systems. J. Biol. Chem. 252, 6438-6442 (1977).

878 56. Einav, T. \& Bloom, J. D. When two are better than one: Modeling the mechanisms of 879 antibody mixtures. Plos Comput Biol 16, e1007830 (2020).

880 57. Gilchuk, P. et al. Analysis of a Therapeutic Antibody Cocktail Reveals Determinants for 881 Cooperative and Broad Ebolavirus Neutralization. Immunity 52, 388-403.e12 (2020).

882 58. Mulangu, S. et al. A Randomized, Controlled Trial of Ebola Virus Disease Therapeutics. New 883 EnglJ Med 381, 2293-2303 (2019).

884 59. Weinreich, D. M. et al. REGN-COV2, a Neutralizing Antibody Cocktail, in Outpatients with 885 Covid-19. New Engl J Medicine 384, NEJMoa2035002 (2020).

886 60. Shah, D. K. \& Betts, A. M. Antibody biodistribution coefficients: inferring tissue 887 concentrations of monoclonal antibodies based on the plasma concentrations in several 888 preclinical species and human. mAbs 5, 297-305 (2013).

889 61. Mudie, D. M. et al. Quantification of Gastrointestinal Liquid Volumes and Distribution 890 Following a $240 \mathrm{~mL}$ Dose of Water in the Fasted State. Mol. Pharmaceutics 11, 3039-3047 891 (2014).

892 62. Schiller, C. et al. Intestinal fluid volumes and transit of dosage forms as assessed by 893 magnetic resonance imaging. Aliment Pharmacol Ther 22, 971-979 (2005).

894 63. Gerding, D. N., Johnson, S., Rupnik, M. \& Aktories, K. Clostridium difficile binary toxin CDT. 895 Gut Microbes 5, 15-27 (2014).

896 64. Studier, F. W. Protein production by auto-induction in high-density shaking cultures. Protein 897 Expres Purif 41, 207-234 (2005).

898 65. Moré, J. J. Numerical Analysis. Lect Notes Math 105-116 (1978) doi:10.1007/bfb0067700.

899 66. Akaike information criterion statistics. vol. 29 (reidel, 1987). 




G-S-G-S-G-G G-S-G



B

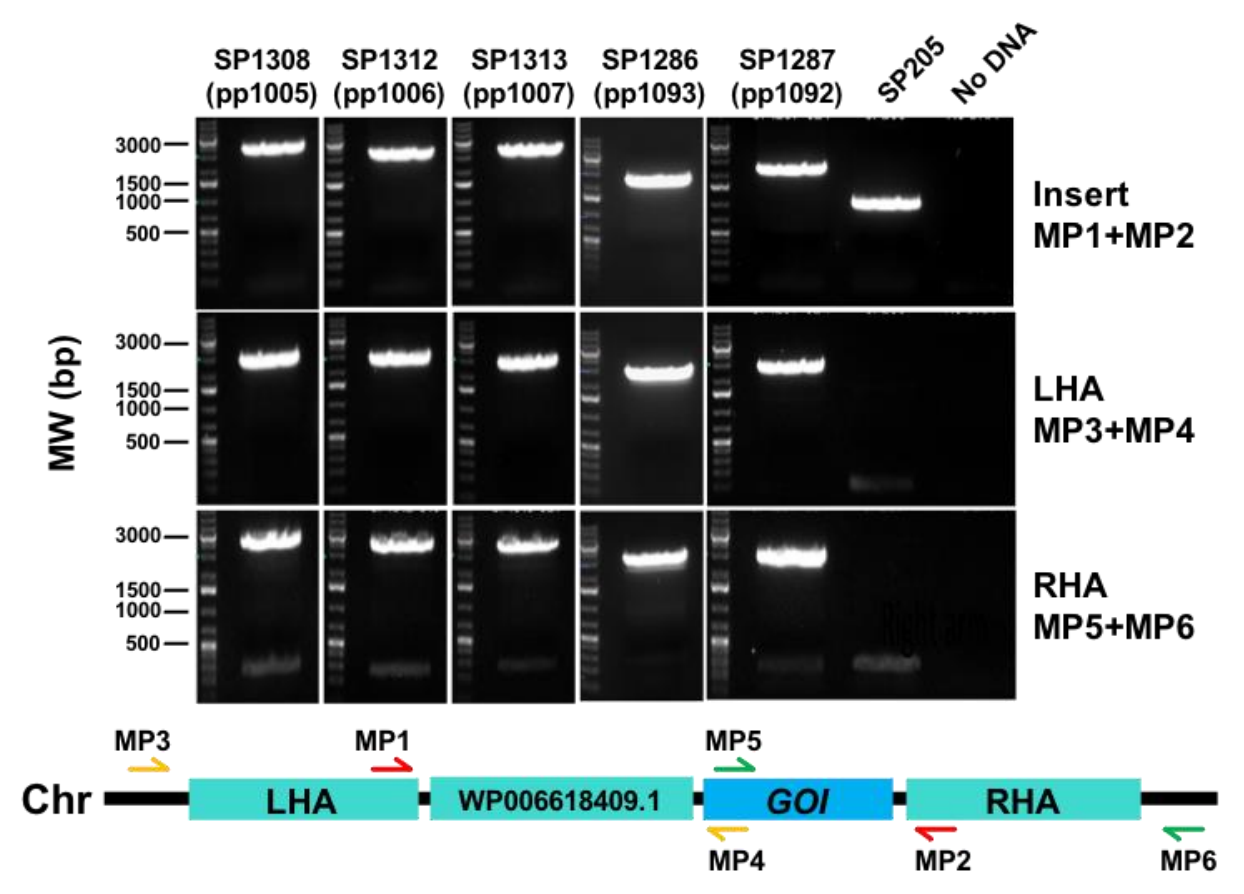

Figure 1. Spirulina strain engineering and characterization. A) Diagram of modified antischematic diagram of the genomic integration locus (WP_006618409.1). PCR was performed with indicated primers to analyze the segregation and specificity of integration for each strain. PCR products with primers MP1/MP2 showed bands of predicted sizes in all strains, and absence of the parental alleles, suggesting the segregation to homozygosity was complete. The primer pairs for amplification of the left homology arm (LHA) and right homology arm (RHA) include one priming site (MP3 and MP6) that is only present in the spirulina genome at the target locus and absent from the donor plasmid. PCR amplification of the LHA and RHA with $\mathrm{MP3/MP4}$ and MP5/MP6, respectively, demonstrated that genomic integration was sitespecific. The DNA sequences from all PCR products were verified to match expectations. 
bioRxiv preprint doi: https://doi.org/10.1101/2021.12.21.473715; this version posted January 14,2022 . The copyright holder for this preprint (which was not certified by peer review) is the author/funder, who has granted bioRxiv a license to display the preprint in perpetuity. It is made available under aCC-BY-NC-ND 4.0 International license.

914 sequencing by comparing reads of the GOls to ten randomly selected native spirulina genes.

915 Dotted line indicates range in which the relative copy numbers were considered equivalent to 916 one.

917 
A

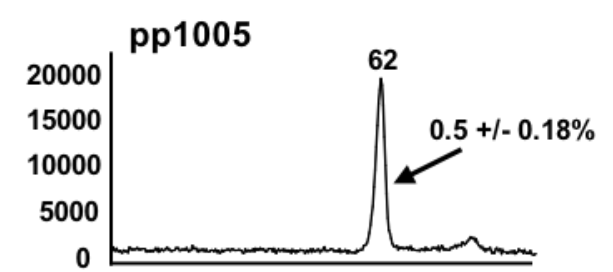

0



pp1007

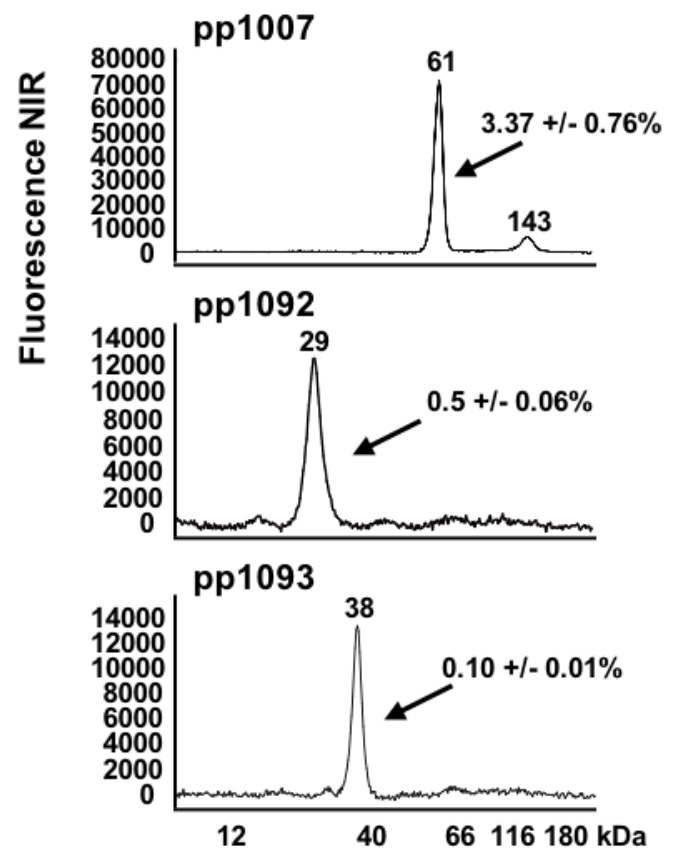

B

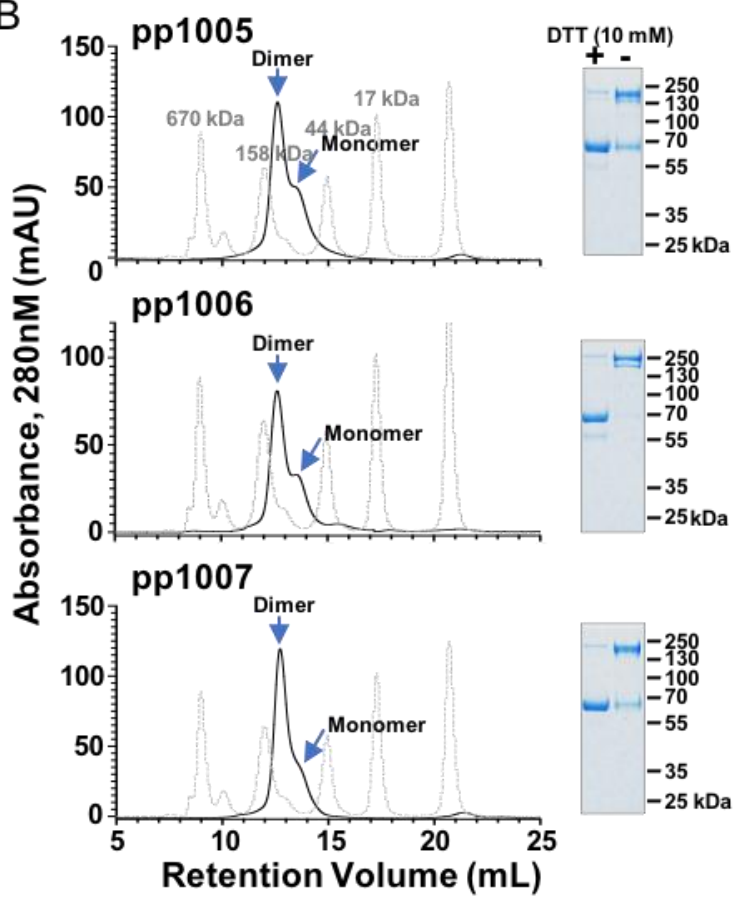

C



Figure 2. Characterization of spirulina-expressed anti-TcdB VHHs and lysins. A) Chromatograms of pp1005, pp1006, pp1007, pp1092, and pp1093 extracted from spirulina biomass and detected under reducing conditions using a capillary electrophoresis-based immunoassay. Soluble protein, as a percentage of washed dry weight, is indicated on each graph. B) Spirulina expressed and purified pp1005, pp1006, and pp1007 in aqueous solution (PBS, pH 7.4) assayed on a Superdex 200 10/300 GL FPLC column. Grey peaks correspond to molecular size standards. The monomeric and dimeric portions of the VHHs are indicated. Disulfide bond formation between monomers was assessed using denaturing gel electrophoresis under non-reducing and reducing (50 mM DTT) conditions. C) Native gel electrophoresis analysis of pp1005 $(1,4)$, pp1006 $(2,5)$, and pp1007 $(3,6)$ under non-reducing or reducing (50 mM DTT) conditions. In the presence of reducing agent the dimeric state of the VHHs was mostly preserved. 
A
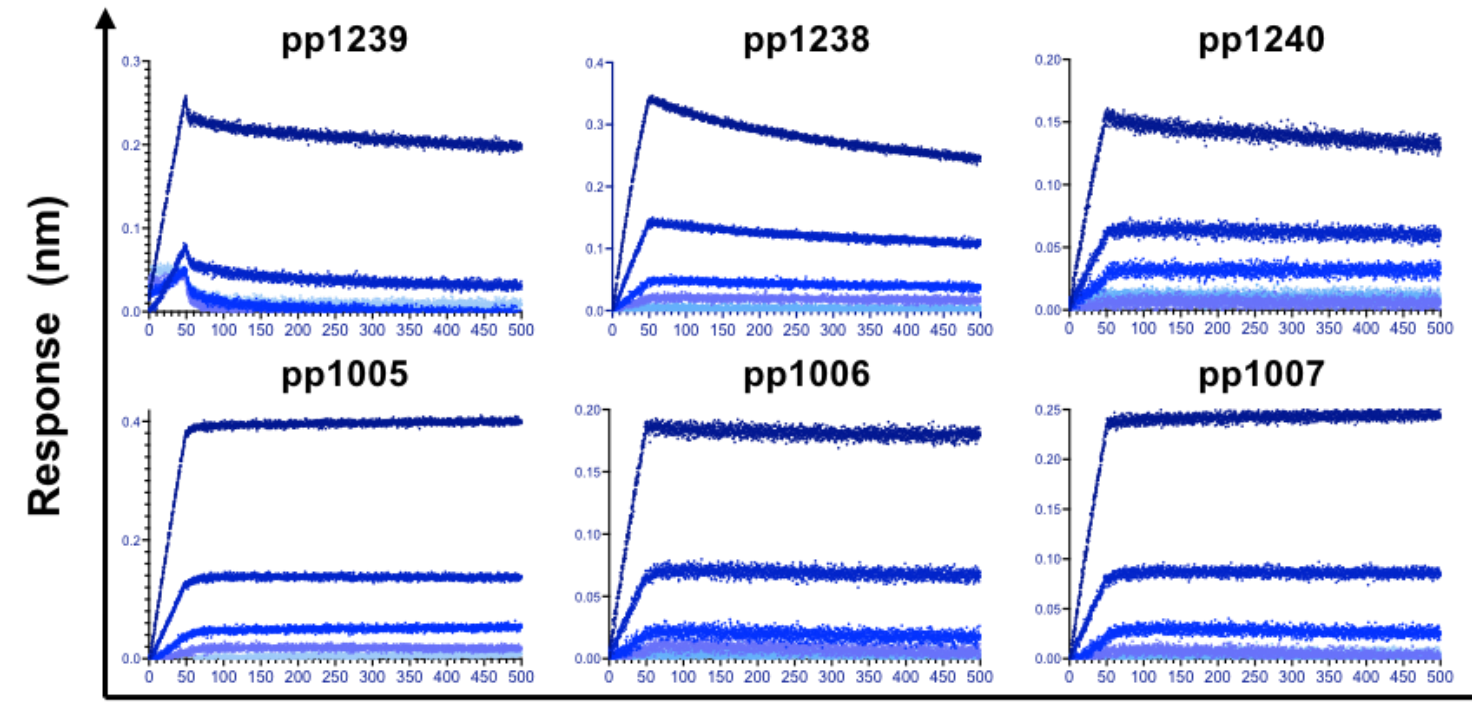

pp1006


Time (s)

B

\begin{tabular}{|c|c|c|c|}
\hline Sample ID & $\mathbf{k}_{\mathrm{D}}(\mathbf{n M})$ & $\mathbf{k}_{\mathrm{a}}(\mathbf{1} / \mathbf{M s})$ & $\mathbf{k}_{\mathrm{d}}(\mathbf{1} / \mathbf{s})$ \\
\hline pp1239 (M) & $2.56(0.02)$ & $1.64 \mathrm{E}+05(0.12)$ & $4.20 \mathrm{E}-04(0.13)$ \\
\hline pp1005 (D) & $0.002(0)$ & $1.16 \mathrm{E}+05(0.11)$ & $2.59 \mathrm{E}-07(0)$ \\
\hline pp1238 (M) & $4.85(0.06)$ & $1.45 \mathrm{E}+05(0.02)$ & $7.02 \mathrm{E}-04(0.02)$ \\
\hline pp1006 (D) & $0.03(0)$ & $7.48 \mathrm{E}+04(1.68)$ & $2.57 \mathrm{E}-06(0)$ \\
\hline pp1240 (M) & $2.09(0.06)$ & $1.26 \mathrm{E}+05(0.04)$ & $2.63 \mathrm{E}-04(0.03)$ \\
\hline pp1007 (D) & $0.58(0.07)$ & $1.54 \mathrm{E}+05(0.17)$ & $8.97 \mathrm{E}-05(0.30)$ \\
\hline
\end{tabular}

Figure 3. Binding affinities and toxin neutralizing activity of TcdB VHHs. A) BLI binding sensorgrams for analysis of binding kinetic for anti-TcdB VHHs as monomers (pp1239, pp1238, and pp1240) and dimers (pp1005, pp1006, and pp1007). B) The rate constants $k_{a}$ and $k_{d}$ were calculated from concentration-dependent responses. The apparent equilibrium binding constant $\left(\mathrm{K}_{\mathrm{D}}\right)$ for each was determined from the rate of association $\left(\mathrm{k}_{\mathrm{a}}\right)$, and rate of dissociation $\left(k_{d}\right)$. Values of $K_{D}, k_{a}$ and $k_{d}$ are indicated in the table ( $M$, monomer; $D$, dimer). 
A

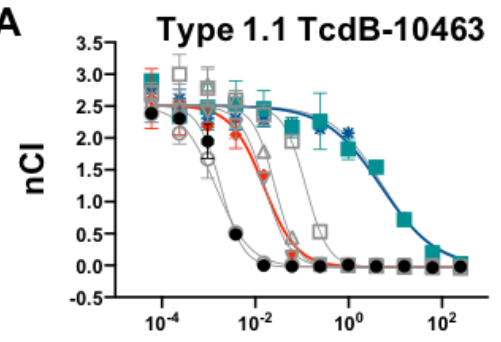

B
Type 3.1 TcdB-1470

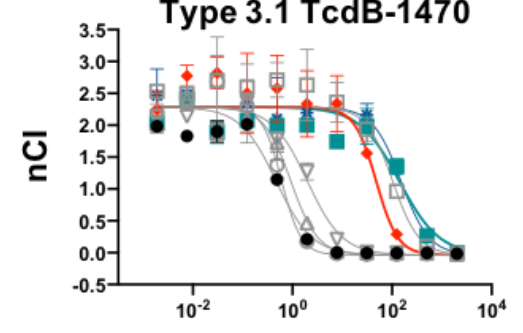

TcdB concentration (pM)
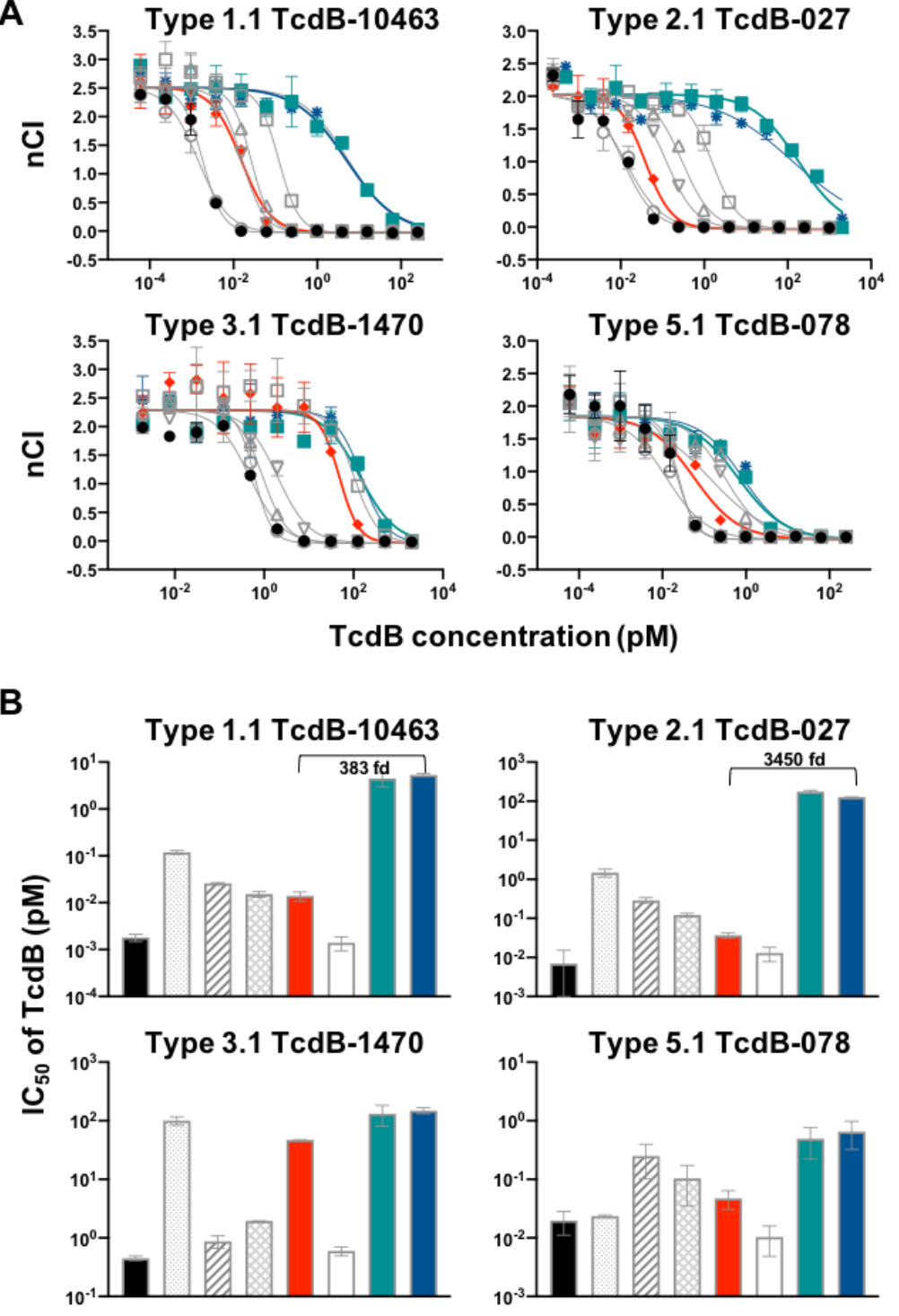

Type 2.1 TcdB-027

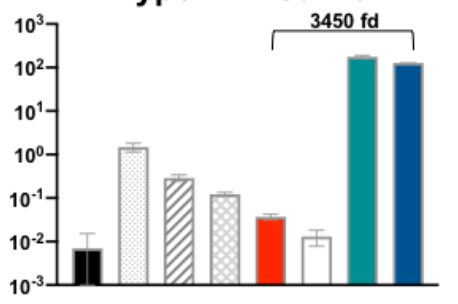

Type 5.1 TcdB-078

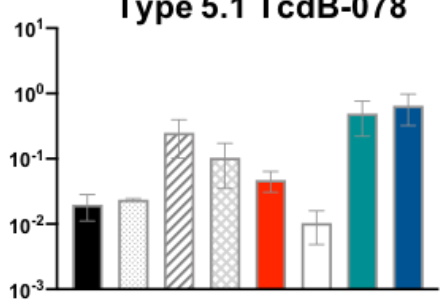

- TcdB alone

曰pp1005, $1.5 \mathrm{nM}$

$\triangle$ pp1006, $1.5 \mathrm{nM}$

$\nabla$ pp1007, $1.5 \mathrm{nM}$

$\leadsto$ bezlotoxumab, 3nM

$\ominus$ pp1092, 1nM

- - pp1005+pp1006+pp1007, 0.5nM of each

*-pp1005+pp1006+pp1007, $0.5 \mathrm{nM}$ of each, +pp1092, 1nM

Figure 4. Comparative neutralization of the five major TcdB types by LMN-201 and bezlotoxumab. A) Neutralizing activity of pp1005, pp1006, pp1007, and the triple cocktail on toxin types 1.1, 2.1, 3.1 and 5.1. $1.5 \mathrm{nM}$ of each dimeric $\mathrm{VHH}$ or the cocktail of all three dimeric VHHs ( $0.5 \mathrm{nM}$ of each) was tested against serially diluted TcdB with Vero cells on the RTCA system. As comparison, $3 \mathrm{nM}$ of bezlotoxumab was similarly evaluated. To rule out interference, the effect of $1 \mathrm{nM}$ lysin on VHH cocktail efficacy was assessed. B) IC 50 of TcdB under treatment was calculated from the data shown in A and compared. 
A

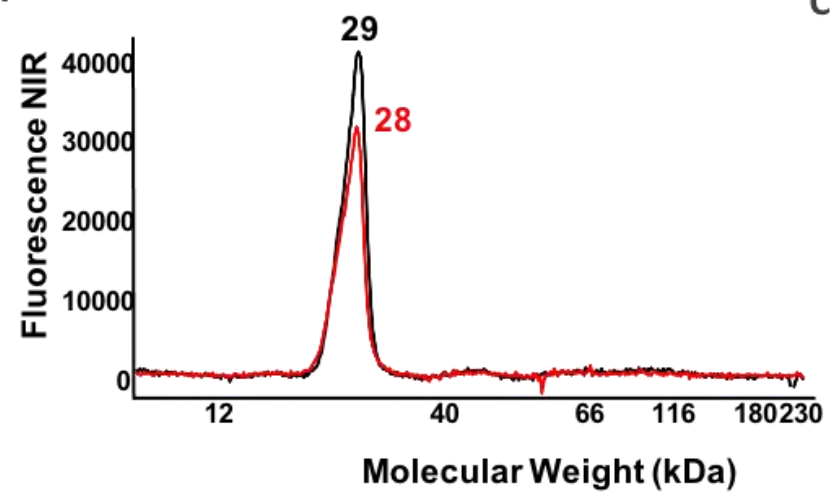

B

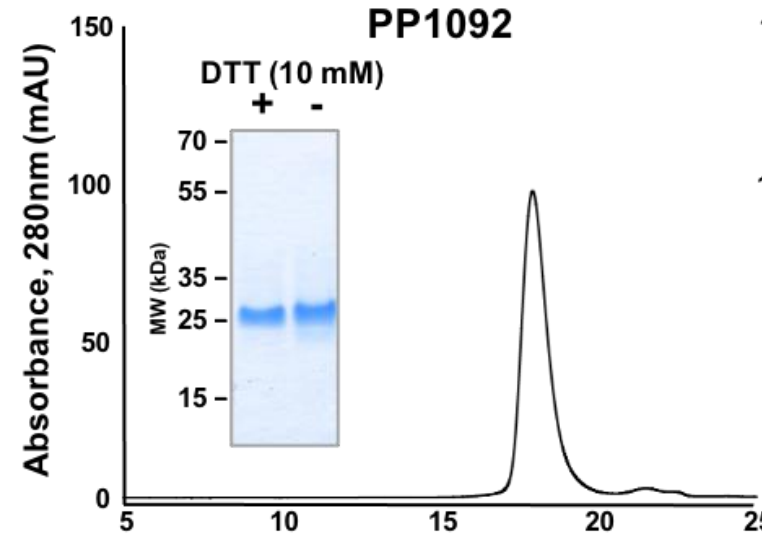

C

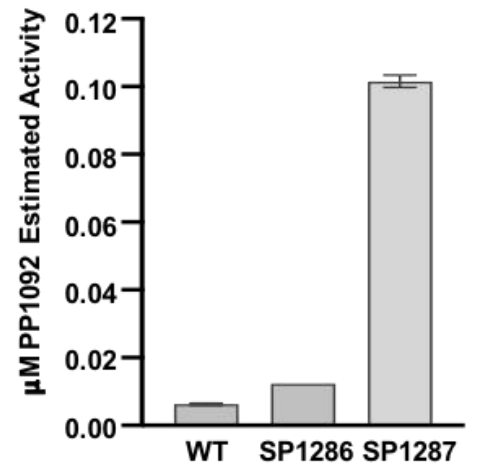

954

955

956

957

958

959

960

961

962

963

964

965

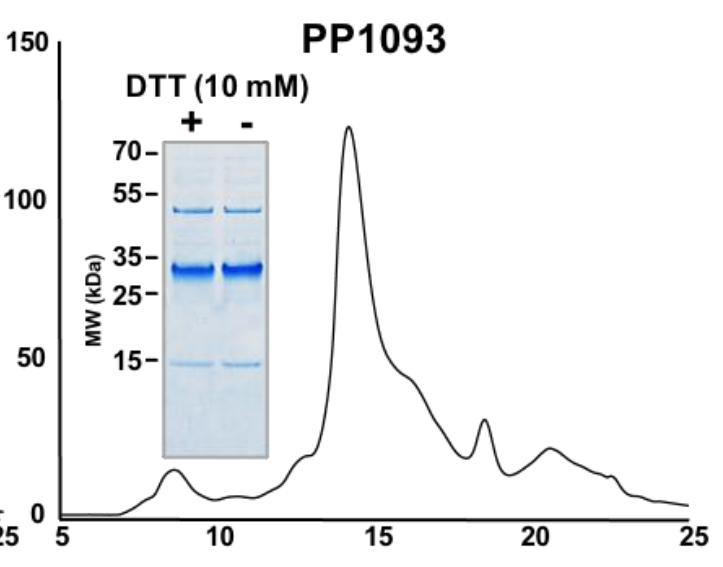

Retention Volume ( $\mathrm{mL}$ )

Figure 5. Characterization of spirulina-expressed anti-C. difficile lysin. A) A chromatogram of pp1092 after total protein extraction (black) and soluble protein extraction (red). More than $75 \%$ of the lysin protein is soluble. B) Spirulina-expressed, purified lysins, pp1092 and pp1093, in aqueous solution (1xPBS, pH 7.4) evaluated by size-exclusion chromatography (Superdex 200 10/300 GL FPLC column). Purified protein samples were also assessed using denaturing gel electrophoresis under non-reducing and reducing (50 mM DTT) conditions. C) Activity of spirulina extracts (wild-type (WT) expressing no lysin, SP1286 expressing pp1093, and SP1287 expressing pp1092) prepared at $2 \mathrm{mg} / \mathrm{mL}$ were compared by measuring the time to reduce turbidity of living $B$. subtilis SL4 cultures by $50 \%$. 

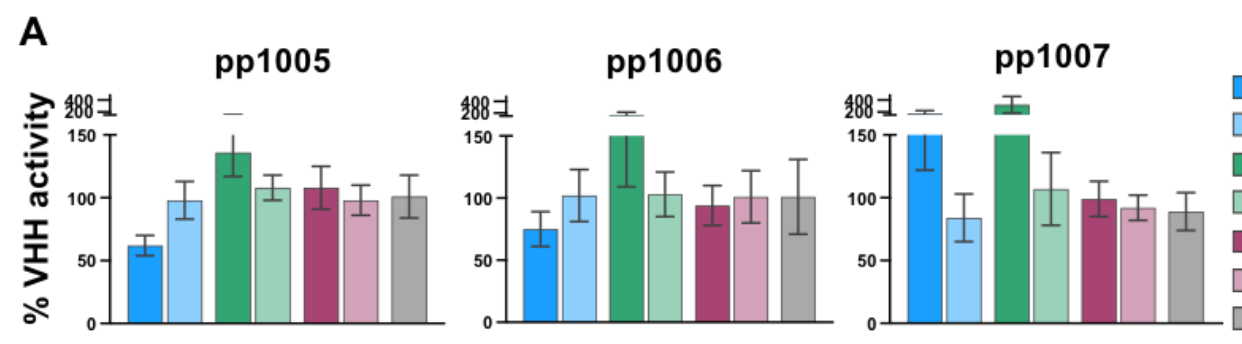

$0.1 \mathrm{mg} / \mathrm{mL}$ trypsin $\square 0.01 \mathrm{mg} / \mathrm{mL}$ trypsin $0.1 \mathrm{mg} / \mathrm{mL}$ chymotrypsin $\square 0.01 \mathrm{mg} / \mathrm{mL}$ chymotrypsin $5 \mathrm{ug} / \mathrm{mL}$ elastase $\square 0.5 \mathrm{ug} / \mathrm{mL}$ elastase

B
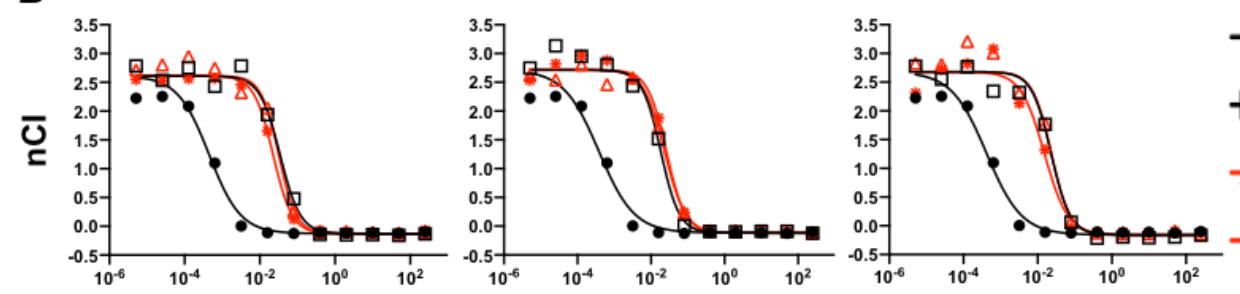

TcdB alone

も VHH, no protease

VHH, $0.1 \mathrm{mg} / \mathrm{mL}$ chymotrypsin (10 min) VHH, $0.1 \mathrm{mg} / \mathrm{mL}$ chymotrypsin (60 min)

TcdB concentration ( $\mathrm{pM}$ )

Figure 6. Stability of anti-TcdB VHHs to gastrointestinal proteases. A) The percentage of TcdBbinding activity of pp1005, pp1006, and pp1007 measured by ELISA after digestion with trypsin $(T)$, chymotrypsin $(C)$, and elastase $(E)$. Two concentrations of each protease were tested to simulate either "fed" (higher concentration) or "fasted" (lower concentration) conditions. Data represent the average of six independent replicates, the error bars representing the standard deviation. B) TcdB-neutralizing activity of protease digested pp1005, pp1006, and pp1007. VHH homodimers treated with chymotrypsin for either 10 or 60 minutes were compared in a cellrounding assay with the corresponding undigested protein. Each was diluted to a fixed concentration (pp1005 was $0.05 \mathrm{nM}$, pp1006 was $5 \mathrm{nM}$, and pp1007 was $5 \mathrm{nM}$ ) and incubated with serially diluted TcdB-10463. Each mixture was assayed for its effect on cell adhesion. After digestion all exhibited neutralizing activities similar to undigested controls. 
A

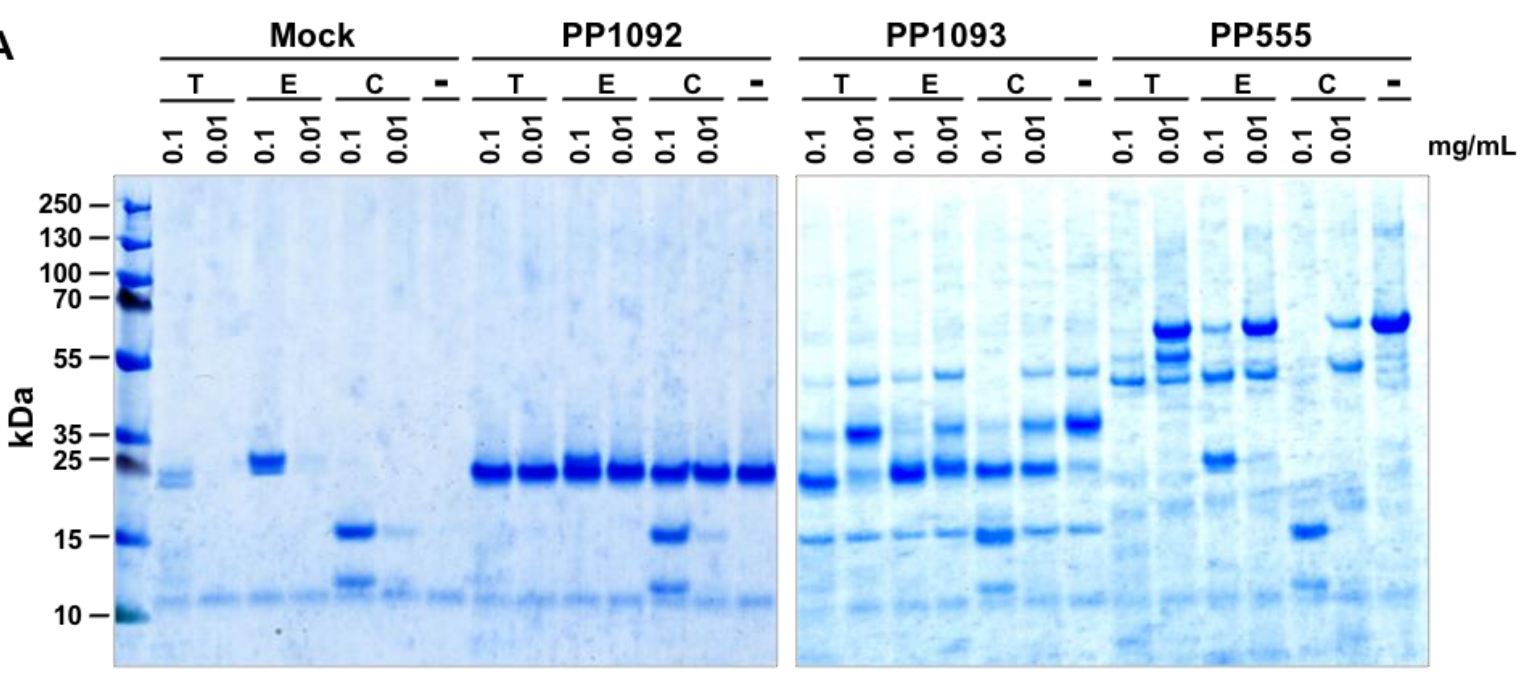

B

C
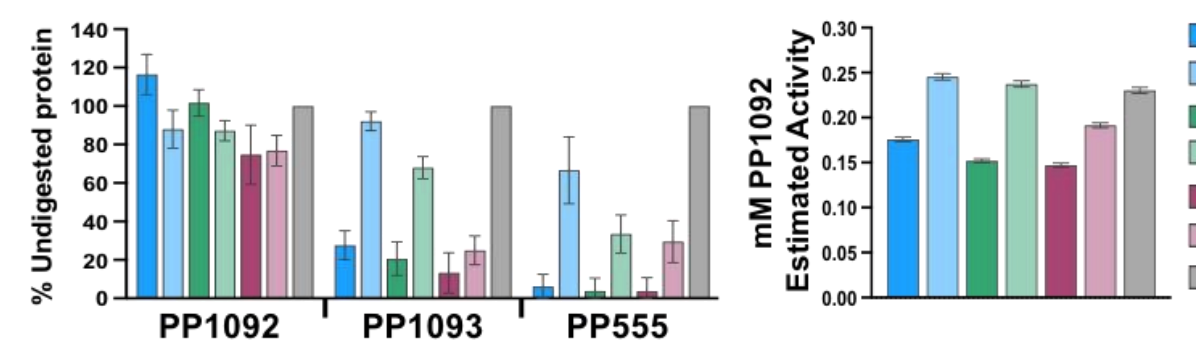

$0.1 \mathrm{mg} / \mathrm{mL}$ trypsin $0.01 \mathrm{mg} / \mathrm{mL}$ trypsin $0.1 \mathrm{mg} / \mathrm{mL}$ chymotrypsin $0.01 \mathrm{mg} / \mathrm{mL}$ chymotrypsin $5 \mathrm{ug} / \mathrm{mL}$ elastase $0.5 \mathrm{ug} / \mathrm{mL}$ elastase No protease

Figure 7. Stability of anti-C. difficile lysin to gastrointestinal proteases. A) Full-length lysin (pp1093, $37 \mathrm{kDa}$ ), its catalytic subdomain (pp1092, $25 \mathrm{kDa}$ ), and a protease sensitive VHH (pp555, $55 \mathrm{kDa}$ ) were digested with trypsin (T), chymotrypsin (C), and elastase (E) at pH 6.4 for 1 hour at $37^{\circ} \mathrm{C}$, quenched with protease inhibitors, the products separated by size using PAGE, and peptides visualized by Coomassie staining. Mock digestions without target proteins were performed to visualize the contribution by the proteases and quenching reagents. B) The image in A (representative of six independent digestions) was quantified by densitometry with error expressed as SEM. C) Activities of pp1092 digests were measured by determining the time to reduce the turbidity of a culture of live $B$. subtilis SL4 by $50 \%$. This value was then plotted against a standard curve of undigested reference lysin to determine the activities of the digested samples compared to undigested reference lysin. The dotted line represents the activity of lysin when undigested. Error bars represent the standard error calculated from three 994 technical replicates. 
A

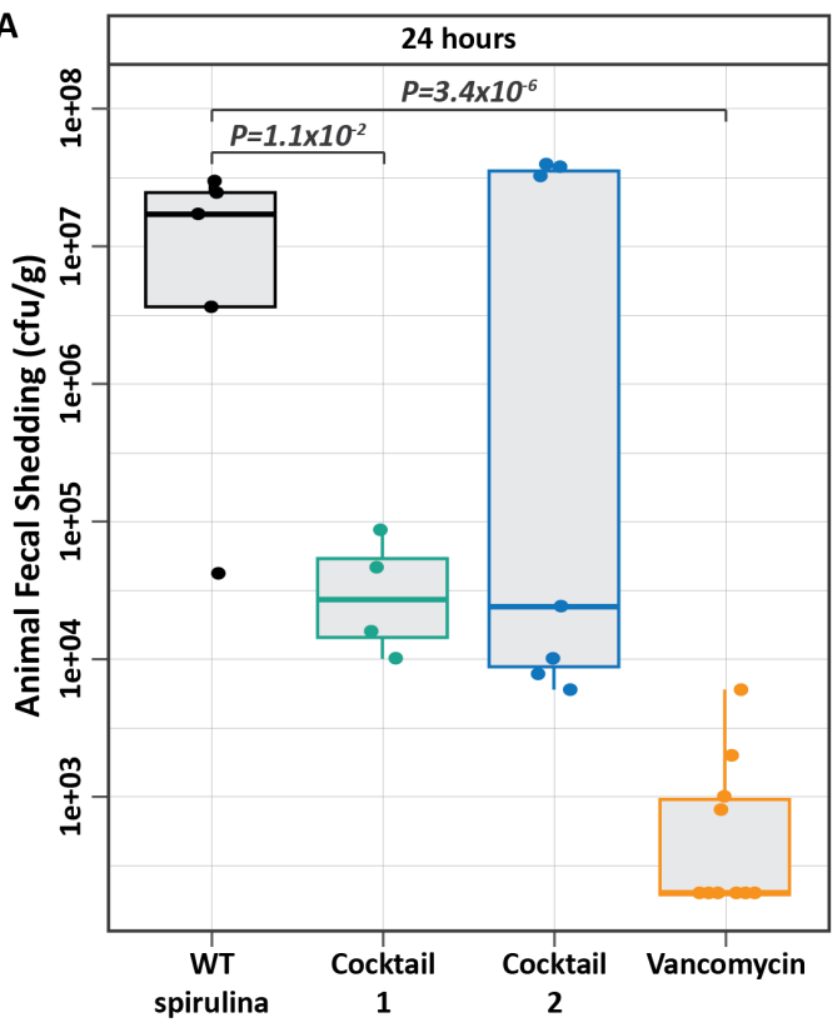

B

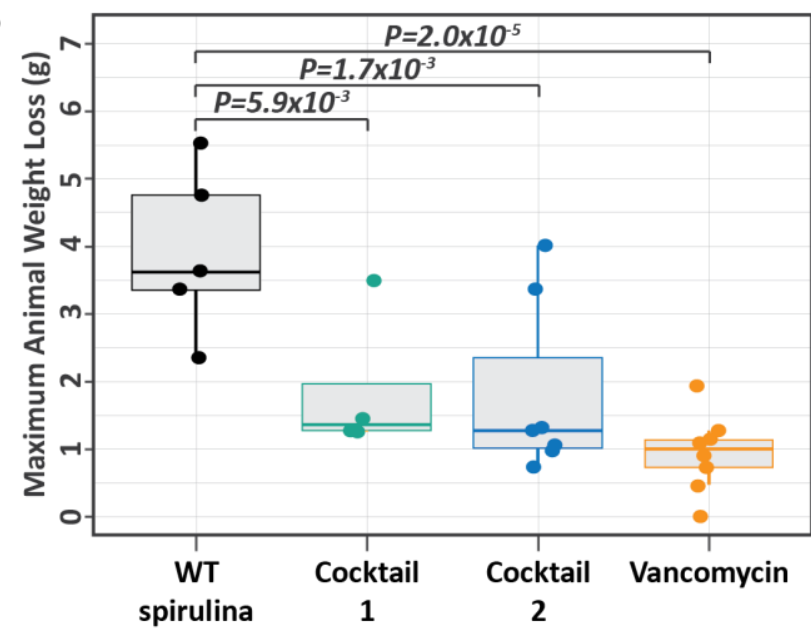

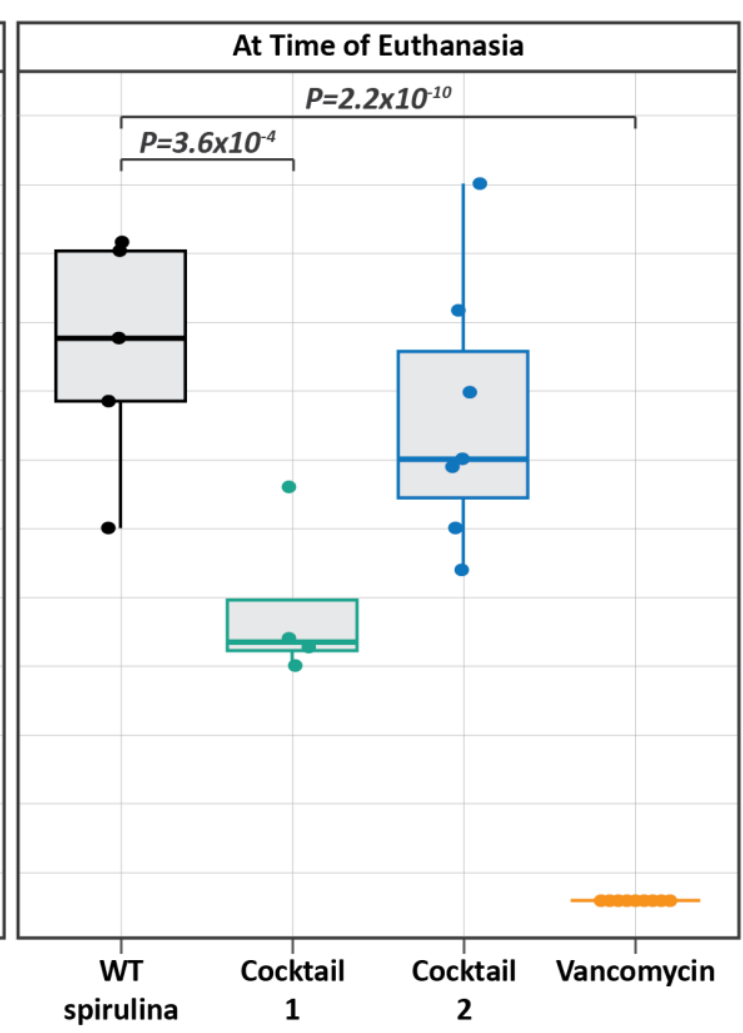

C

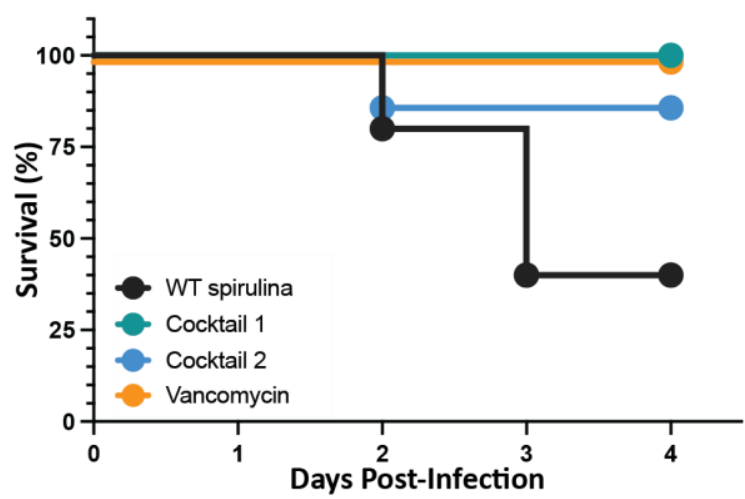

Figure 8. Mouse challenge study. Shown are A) fecal spore counts 24 hours after infection and at the time of euthanasia; B) maximum weight loss for individuals in each group; and C) KaplanMeier representation of survival. Individuals were euthanized upon losing $\geq 15 \%$ of their initial weight. Individuals in panels $A$ and $B$ are represented as a point and the group median, interquartile range (IQR) and $1.5 * I Q R$ as boxplots. A) $\log _{10}$ fecal shedding data were fit with a linear model with group as a predictor per time point. Treatment group is a significant predictor $(p<0.05)$ of shedding relative to the wild-type spirulina group (control/intercept) for Cocktail 1 and vancomycin. Cocktail 2 fails significance testing ( $p^{\sim 0.1}$ and $p^{\sim 0.25}$ ). Statistic reports the $t$ ratio based on a Student $t$ reference distribution, and $p$-value reports the two-tailed $p$-value corresponding to the $t$ ratio based on the Student $t$ reference distribution. $B$ ) Maximum animal 
1007 weight loss was fit with a linear model with treatment as predictors of weight loss. Treatment 1008 group is a significant predictor $(p<0.05)$ of animal weight loss relative to the wild-type spirulina 1009 group (control). C) Kaplan-Meier survival curves for mice infected with $10^{5} \mathrm{C}$. difficile spores and 1010 treated with vancomycin (orange, $n=10$ ), wild-type spirulina (black, $n=5$ ), spirulina therapeutic 1011 Cocktail 1 (teal, $n=5$ ) or Cocktail 2 (blue, $n=7$ ). Survival rates were compared to treatment with 1012 wild-type spirulina by log-rank test. Significance was observed with vancomycin treatment $1013(p=0.0068)$, borderline significance was observed with spirulina Cocktail $1(p=0.080)$ and 1014 Cocktail $2(p=0.14)$ treatment. 

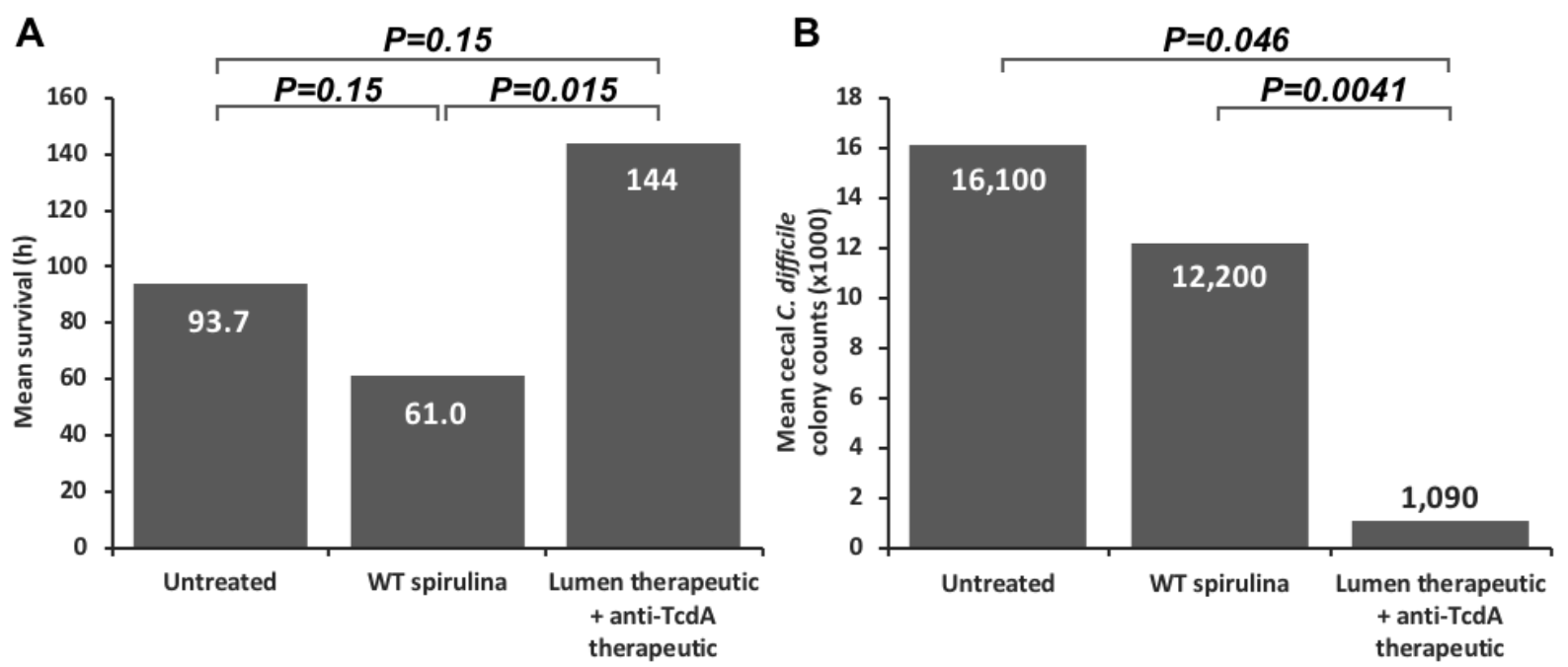

Figure 9. Hamster challenge trial. A) The Lumen therapeutic prolonged survival times when compared to the untreated and group administered wild-type spirulina. Clindamycin-treated Golden Syrian hamsters were administered phosphate-buffered saline, wild-type spirulina, or the Lumen therapeutic two times a day starting one day before infection with $C$. difficile $(15,000$ spores) and continuing until they reached their humane endpoints or the end of the study (14 days post-infection). The mean survival times were compared using a Mann-Whitney test with continuity correction, $90 \%$ confidence level, one-sided test. B) Lumen therapeutic decreased $C$. difficile cecal colonization. Cecal contents were collected after animals reached their humane endpoints or the termination date. $C$. difficile colony counts were quantified by comparing RT-PCR measurements of extracted DNA from these samples as compared to a standard curve generated by DNA extracted from vegetative $C$. difficile cells. Fewer $C$. difficile cells colonized the cecum in hamsters treated with the Lumen therapeutic than in wild-type spirulina-treated or untreated groups. Wilcoxon rank sum test (Mann-Whiney test) with continuity correction, $90 \%$ confidence level, one-sided test. 
1033 1034 1035 1036 1037 1038 1039 1040

\begin{tabular}{|c|c|c|c|c|c|}
\hline Parameter & Estimate & Std. error & p-value & $2.5 \% \mathrm{Cl}$ & $97.5 \% \mathrm{Cl}$ \\
\hline TcdB IC 50 (fM) & 1.268 & $6.57 \mathrm{E}-05$ & $2.24 \mathrm{E}-52$ & 0.001138 & 0.001397 \\
\hline Hill $^{\text {TcdB }}$ & 1.495 & 0.07091 & $1.35 \mathrm{E}-58$ & 1.355 & 1.634 \\
\hline $\mathrm{EC}_{50} \mathrm{pp} 1005^{\mathrm{p}}(\mathrm{pM})$ & 21.44 & 0.002423 & $1.28 \mathrm{E}-16$ & 0.01667 & 0.02622 \\
\hline $\mathrm{Hill}^{\mathrm{pp} 1005}(-)$ & 1.813 & 0.1245 & $1.12 \mathrm{E}-35$ & 1.567 & 2.058 \\
\hline$E_{\max }^{p p 1005}$ (fold) & 45.25 & 3.076 & $3.20 \mathrm{E}-36$ & 39.2 & 51.31 \\
\hline$E C_{50}{ }^{p p 1006}(p M)$ & 54.13 & 0.003761 & $4.25 E-35$ & 0.04673 & 0.06154 \\
\hline$E C_{50}{ }^{p p 1007}(p M)$ & 69.26 & 0.004852 & $1.11 \mathrm{E}-34$ & 0.05971 & 0.07882 \\
\hline Bottom (assay units) & -0.01326 & 0.006172 & 0.0326 & -0.02541 & -0.001107 \\
\hline Top (assay units) & 0.9289 & 0.01145 & 3.39E-189 & 0.9064 & 0.9515 \\
\hline
\end{tabular}

Table 1. $\mathrm{EC}_{50}$ values for pp1005, pp1006, and pp1007. Independent model estimates from analysis of the overall data set. TcdB1 acts as an inhibitor of cellular adhesion with femtomolar potency $\left(I C_{50}, H\right)$. pp1005, pp1006, and pp1007 act to inhibit TcdB1 activity, shifting TcdB1 potency 2-fold at 10 's of picomolar potency $\left(\mathrm{EC}_{50}{ }^{\mathrm{pp} 1005}, \mathrm{EC}_{50}{ }^{\mathrm{pp} 1006}, \mathrm{EC}_{50}{ }^{\mathrm{pp} 1007}\right)$. pp1005 activity saturates at around 50 -fold $\left(E_{\max }{ }^{\text {pp1005 }}\right.$, Hill $\left.{ }^{p p 1005}\right)$ while the somewhat less potent pp1006 and pp1007 do not saturate. 